\title{
Grain boundaries and interfaces in slip transfer
}

\author{
T.R. Bieler ${ }^{\mathrm{a}, *}$, P. Eisenlohr ${ }^{\mathrm{a}, \mathrm{b}}$, C. Zhang ${ }^{\mathrm{a}}$, H. Phukan ${ }^{\mathrm{a}}$, M.A. Crimp ${ }^{\mathrm{a}}$ \\ ${ }^{a}$ Chemical Engineering and Materials Science, Michigan State University, East Lansing, MI 48824, USA \\ ${ }^{b}$ Max-Planck-Institut für Eisenforschung, 40237 Düsseldorf, Germany
}

\begin{abstract}
The effect of slip transfer on heterogeneous deformation of polycrystals has been a topic of recurring interest, as this process can either lead to the nucleation of damage, or prevent nucleation of damage. This paper examines recent experimental characterization of slip transfer in tantalum, TiAl, and Ti alloys. The methods used to analyze and assess evidence for the occurrence of slip transfer are discussed. Comparisons between a characterized and simulated patch of microstructure are used to illustrate synergy that leads to new insights that cannot arise with either approach alone.
\end{abstract}

Keywords: grain boundary, slip transfer, titanium, tantalum, mechanical twinning, damage nucleation, geometrically necessary dislocations

\section{Introduction}

Heterogeneous deformation of metallic polycrystals has been an active topic of research in the past decade, as the community has come to realize that deformation not only varies amongst grains in a polycrystal, but significant gradients in deformation occur within grains as well. This realization has come because of new tools that are able to characterize this phenomenon, such as EBSP mapping (a.k.a. Orientation Imaging Microscopy $^{\mathrm{TM}}$, or OIM) and 3D synchrotron X-ray diffraction that have been important enabling methodologies. These tools have permitted to examine finer details of heterogeneous deformation $[1,2]$. Some of the clearest evidence for the heterogeneity of slip is evident in characterization of deformed oligocrystals with nominally columnar grains. These samples are especially useful for comparing material modeling strategies with experiments, because they facilitate generation of meshes for crystal plasticity modeling [3-5] from surface information alone.

Prior to this realization, modeling the deformation of polycrystals used statistical models based on the Taylor factor (which assumes that all grains strain uniformly), leading to varying stresses that are averaged (homogenized) out. This approach is useful for generating material models used for design and prediction of macroscale deformation. However, the plastic anisotropy of single crystal deformation results in heterogeneous boundary conditions and consequently heterogeneous deformation among and within the grains of a polycrystal (thus violating the assumption of the Taylor factor), resulting in, for instance, surface rumpling (a.k.a. orange-peel effect) and stress or strain concentrations that precede and may facilitate development of a critical flaw. Statistical crystal plasticity models, such

*Corresponding author. Phone: +1 517 353-9767, fax: +1 517 432-1105
Email address: bieler@egr.msu.edu (T.R. Bieler) as the visco-plastic self-consistent model, improved on the Taylor assumption by allowing individual grains to deform differently within a homogeneous medium representing the average material behavior and requiring only the average strain among the orientations to match the imposed deformation [6]. Nevertheless, such models still assume uniform strain within each grain (unless more sophisticated multi-orientation schemes are used [7-9]).

Even with these improvements, it has been generally accepted that simulations tend to show a sharper distribution of orientations than is typically measured. This lack of agreement can be ascribed to the fact that a grain within a polycrystal experiences spatially heterogeneous boundary conditions resulting from and depending on the different plastic activity in its surrounding grains. Consequently, different orientations develop within a given grain. To capture this effect in computational models of microstructure patches, representative volume elements are computationally deformed with crystal plasticity based constitutive models that allow strains to vary within a given grain. One way to interpret the strain heterogeneity is to compute local Taylor factors based upon the local kinematics [3, 10-13]. Assessment of local Taylor factors has also been effective in rationalizing the origins of pore nucleation; for example, voids are more likely to develop in regions with both hard and soft orientations, than in regions that are mostly hard. Void development is also more likely at grain boundaries between soft orientations where differential strains from predominant slip systems cause incompatible shape changes $[14,15]$.

What is missing in above treatments is the explicit consideration of grain boundary properties, such as whether a grain boundary is intrinsically strong or weak, if the process of dislocation interactions with the boundary weakens it, or if features such as ledges in the grain boundary plane facilitate dislocation nucleation that enables localized stress relaxation [16-18]. Introduction of grain boundary properties in models has a strong 


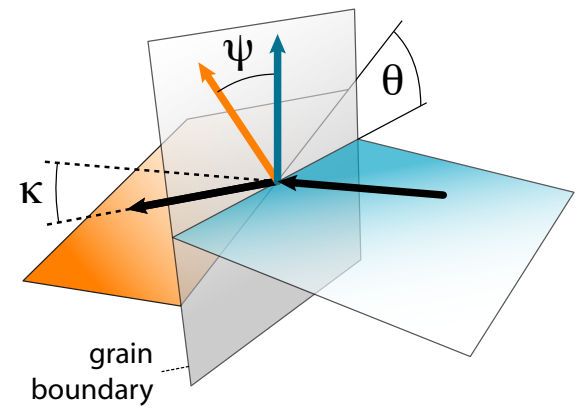

Figure 1: Geometry of slip transfer across a grain boundary. Horizontal (orange and blue) planes signify slip or twinning planes on either side of the boundary. $\kappa$ is the angle between slip directions, $\psi$ is the angle between plane normals, and $\theta$ is the angle between plane traces on the boundary.

influence on strain evolution in polycrystal simulations, particularly when cracking is modeled [19-22]. Given this reality, there is a current discussion about how grain boundary character (however defined) influences plasticity in the two neighboring grains. As grain boundaries are common locations for damage nucleation, this ongoing discussion may lead to significant contributions to our ability to predict damage (and its variability) in a deterministic way.

The quantitative study of slip interactions at grain boundaries started about 60 years ago, with the investigations of slip transfer across boundaries in bi-crystals by Livingston and Chalmers [23]. The geometry of slip transfer between two slip systems on either side of a boundary is often defined in Fig. 1 by the three angles $\kappa$ (the angle between slip vectors), $\psi$ (the angle between slip plane normals), and $\theta$ (the angle between the two slip plane intersections with the grain boundary plane). Though Livingston and Chalmers proposed a quantitative rationale for slip transfer to occur, a later effort that analyzed slip transfer using transmission electron microscopy (TEM) in an austerity stainless steel (a collaboration of Clark, Wagoner, Shen, Lee, Robertson, and Birnbaum [24-26]) showed that slip transfer could be predicted when the product $M=\cos \kappa \cos \theta$ was maximized. This (LRB) criterion is explained as follows:

1. The angle $\theta$ between the slip plane traces on the grain boundary plane must be a minimum (i.e. minimize $\theta$ ), minimizing the angle between the tangent vectors of the incoming and outgoing dislocations.

2. The magnitude of the Burgers vector of the dislocation left in the grain boundary must be a minimum (i.e. minimize $\kappa)$.

The importance of the resolved shear stress on the outgoing slip system was identified by this group, but this is not an integral part of a slip transfer parameter. Furthermore, this factor does not take into account the possibility of a variable slip plane (cross slip) near the boundary. A similar effort was made to parametrize slip transfer between grains in TiAl by Luster and Morris [27] using related ideas, defining $m^{\prime}=\cos \kappa \cos \psi$, which was able to account for observations of slip transfer in TEM specimens.
The notation of these slip transfer variables is a bit unfortunate, as $M$ is often used for the Taylor factor, and $m$ for the Schmid factor, both of which involve the stress tensor, whereas the $m^{\prime}$ and the $M$ parameters are strictly geometrical. Hereafter, we will refer to $M$ simply as LRB. In practice, LRB is difficult to evaluate in experiments, because the inclination of the grain boundary plane is not easily obtained in a nondestructive manner. The $m^{\prime}$ parameter is more convenient for non-destructive experimental work because it can be evaluated using EBSP (OIM) data sets on initially polished surfaces. Both $m^{\prime}$ and LRB can be easily evaluated in computational experiments (e.g. [28, 29]). At present, it is arguable whether the predictive power of $m^{\prime}$ or LRB is more effective.

There is further ambiguity in the use of either $m^{\prime}$ or LRB as there are typically more than one pair of slip systems active in the grain boundary regions of deforming polycrystals. 3D tomographic characterizations of actual boundaries show complex arrangements of dislocations on different slip systems, which implies that multiple slip processes take place and interact with each other and the boundary [30, 31]. Multiple slip systems on either side of the boundary leads to multiple meaningful values of $m^{\prime}$ or LRB; there are as many values as the number of slip systems squared. Pairs of slip systems with high Schmid factors have relatively low values of $m^{\prime}$ or LRB. Furthermore, the geometrical description presented so far does not consider the effects of residual dislocation content left in the boundary, which requires assessment of the incoming and outgoing dislocation Burgers vector sums $[30,31]$. This then leads to a continuum of cases to consider with regard to slip transfer [32], with exemplary cases identified below:

1. the grain boundary acts as an impenetrable boundary that does not allow shear to be transferred to the neighboring grain, leading to a dislocation pileup and local stress concentration. This results in accumulation of geometrically necessary dislocations (GNDs) and local lattice curvature [33] in order to maintain boundary continuity;

2. the boundary is not impenetrable, and slip in one grain can progress into the next grain with some degree of continuity (leaving residual boundary dislocations), and perhaps only partial ability to accommodate a shape change imposed by a neighboring grain;

3 . the boundary is nearly transparent to dislocations on specific activated slip systems, and (near) perfect transmission can occur, i.e. little deformation resistance (e.g. Ilines, low-R boundaries [34]) or low-angle boundaries $[33,35,36])$. In a finite element mesh grain boundary, there is typically no slip resistance across the boundary unless it is introduced into the model.

Note that any given boundary characteristic identified above depends on the activated slip systems - the same boundary could fall into a different category with a different loading condition.

Clearly, the role of the stress tensor is important, as it identifies which slip systems are active because $m^{\prime}$ or LRB values for inactive slip systems are irrelevant. This implies that it is important to meaningfully identify the relevant state of stress in the region adjacent to the grain boundary. Observations of 
heterogeneous deformation show that even when a polycrystal is deformed in uniaxial tension, non-uniform deformation must lead to deviations from the uniaxial stress state, and this further affects which slip systems have the greatest driving force for activation. This implies that the driving force for slip activation will vary from the boundary to the interior of the grain. Spatially resolved crystal plasticity simulations provide the most convenient method to identify gradients in stress tensors, as physical measurements of local stress tensors are experimentally challenging.

Such details related to slip transfer are averaged out with regard to predicting overall homogenized material deformation, but these details are significant for identifying the processes that precede damage nucleation. The prediction of damage nucleation is a longstanding grand challenge in metallurgy, because damage nucleation coupled with propagation ultimately defines the useful lifetime of a stressed component. It is possible for single damage nucleation site to grow into a critical crack. In other cases, multiple damage nucleation sites may link up to form a critical crack. Therefore, the spatial arrangement of damaged sites and the evolving local stress state may be the basis for predicting the development of a critical crack. There are many examples of modeling the process that links damaged sites and establishment of a critical crack, with varying degrees of detail involving grain boundary energy and identifying the locus of concentrated slip between damage sites, but such models can only be effective if the 3D spatial distribution of damaged sites can be predicted with confidence. The two processes of damage nucleation and coalescence then control the origin of variability, which is a crucial design constraint at the component level, as designs need to anticipate worst case failure conditions. Consequently, establishing criteria for defining the origin of damage and its distribution within the microstructure is an enabling capability and a critical component of integrated computational materials engineering goals. The detailed mechanisms of damage nucleation hold the key to understanding the source of variability in component lifetimes.

To illustrate progress toward the ability to identify vulnerable grain boundaries in the context of activated slip, several examples of investigations in different materials will be summarized in this paper, starting with pure tantalum, which provides a way to introduce many important analytical subtleties that need to be considered in developing models for damage nucleation. Investigation of cubic metals can be rather challenging, due to the large number of slip systems that can be activated with common slip planes and directions, making identification of these systems difficult in experiments. Thus, the remainder of the examples presented will be for non-cubic metals where the number of activated slip systems is much more limited, which makes it easier to analyze and extract insightful details. Damage nucleation due to twin-grain boundary interaction in TiAl is described next, where a fracture initiation parameter was identified that is based upon slip transfer ideas. Several examples of slip transfer in commercial purity titanium and titanium alloys will then be considered based upon salient points from prior papers and some new results that are published for the first time here. Comparisons with related studies will be made to identify similarities and differences, to provide insights and identify opportunities for future study.

\section{Experimental description}

The majority of the samples examined were nominally $2.5 \times 3 \times 25-30 \mathrm{~mm}^{3}$ four-point bending beams, where the tensile surface of the beam was carefully polished and characterized using OIM prior to deformation. In all cases, the bending was done with a manually adjusted jig, and no stress was measured. Bending samples were considered advantageous to tensile specimens, because they put the maximum stress on the surface that can be microscopically characterized. In-situ tensile deformation in scanning electron microscopy has also been carried out using pre-polished samples with a gage length of $10 \mathrm{~mm}$ and a $2 \times 3 \mathrm{~mm}^{2}$ cross section. The details of sample preparation and deformation are provided in [37-41].

Initial characterization was conducted on various electron microscopes using TSL OIM data acquisition systems. ${ }^{1}$ Data were typically post-processed to remove very small grains so that "reconstructed boundaries" files could be extracted. These data were used to compute slip transfer parameters. A MATLAB code was written to provide a means to visualize slip planes, plane traces, and Burgers vectors within a grain using a unit cell, and to compute $m^{\prime}$ parameters. This code has been developed further with a graphical user interface and will be available as a portion of the DAMASK software suite [43].

The subsurface nature of several of the samples was investigated using two beamlines of the Advanced Photon Source at Argonne National Laboratory. Bend and tensile samples initially characterized using OIM were investigated using differential aperture X-ray microscopy (DAXM) in beamline 34-IDE [44] to examine subsurface grain shapes and stress tensors in samples that were unloaded. In-situ tensile deformation 3D $\mathrm{X}$-ray tomographic analysis was carried out on beamline 1-ID using an annealed grade 1 commercial purity titanium sample with a $80 \mu \mathrm{m}$ grain size, gage length of $5 \mathrm{~mm}$, and cross section of $1 \times 1 \mathrm{~mm}^{2}$ (further details are provided in [45]).

\section{Case Studies}

\subsection{Tantalum}

It might be expected that when $m^{\prime}$ is small, strain transfer across grain boundaries would be difficult as the slip systems on either side of the boundary are poorly aligned. With poor alignment, dislocations may pile up near the boundary, resulting in a high density of GNDs. These GNDs will cause local lattice

\footnotetext{
${ }^{1}$ Of great importance for this work is the correct identification of the Euler angle coordinate system. The calibration procedures for some EBSD systems introduce a $180^{\circ}$ rotation ambiguity in the first Bunge [42] Euler angle $\varphi_{1}$. Accurate analysis of twinning requires this ambiguity to be resolved. While surface trace orientations are not affected by this ambiguity, the derived sense of shear associated with mechanical twinning could be opposite to the only physically possible twinning direction, i.e., appear as anti-twinning [32]. This problem has been addressed in recent EDAX/TSL releases of the OIM acquisition software.
} 


\begin{tabular}{cccccccc}
\hline$\downarrow 82 \& \overrightarrow{55}$ & 0.50 & 0.46 & 0.40 & 0.40 & 0.38 & 0.31 & 0.29 \\
0.46 & 0.17 & 0.07 & 0.24 & 0.23 & 0.08 & 0.31 & 0.06 \\
0.43 & 0.29 & 0.17 & 0.33 & 0.00 & 0.10 & 0.11 & 0.00 \\
0.40 & 0.58 & $\underline{0.67}$ & 0.34 & 0.03 & 0.07 & 0.01 & 0.58 \\
0.38 & 0.13 & 0.05 & 0.18 & $\underline{\mathbf{0 . 9 3}}$ & $\mathbf{0 . 9 7}$ & $\mathbf{0 . 6 4}$ & 0.05 \\
0.37 & 0.02 & 0.05 & 0.08 & 0.39 & 0.25 & 0.43 & 0.11 \\
0.37 & 0.06 & 0.10 & 0.20 & $\mathbf{0 . 6 4}$ & $\mathbf{0 . 9 3}$ & 0.19 & 0.23 \\
0.35 & $\mathbf{0 . 7 4}$ & $\mathbf{0 . 6 7}$ & $\mathbf{0 . 6 2}$ & 0.03 & 0.02 & 0.03 & 0.42 \\
\hline
\end{tabular}

Table 1: Evaluation of $m_{3 m}^{\prime}$ based upon sorted Schmid factors listed in first row for grain 55 and first column for grain 82 $\left(\left\{\begin{array}{lll}1 & 1 & 0\end{array}\right\}\right.$ slip is indicated in gold; $\left\{\begin{array}{lll}1 & 1 & 2\end{array}\right\}$ slip is indicated in purple).

curvature that can be quantified using the OIM local average misorientation (LAM). ${ }^{2}$

In order to examine the relationships between activated slip systems, slip transfer, lattice curvature, and $m^{\prime}$, a polycrystalline sheet of recrystallized pure Ta [46] with a grain size of about $80 \mu \mathrm{m}$ and thickness of $1.3 \mathrm{~mm}$ was studied. Two samples from the same material were incrementally bent to impose tensile strains in the rolling (RD) and transverse (TD) directions, respectively. While the samples were loaded, OIM measurements were made over an area of about $1 \mathrm{~mm}^{2}$. A local average strain was measured using SEM images taken before and after deformation to track distances between selected triple points. Figure 2 compares orientation maps, LAM maps, and modified $m^{\prime}$ maps described in detail below.

It is clear that the spatial distribution of regions of high LAM differs between the rolling and transverse direction maps (RD and TD). The RD deformed sample has extensive regions in grain interiors where there is very low GND content, while the TD sample has a much more uniform level of GND content. The grain boundaries where GND accumulation is found are at various inclinations with respect to the horizontal tensile axis in the TD deformed sample. In contrast, there is a tendency for boundaries that are perpendicular to the tensile direction to accumulate GNDs in the RD sample.

The central part of Fig. 3 shows an enlarged patch of deformed microstructure indicated by the box in Fig. 2(a) after deformation that illustrates significant heterogeneous deformation. In some grains, well-defined slip lines are evident, while in others, the slip activity is not as obvious. Some show single slip and others multiple slip (such as grain 83). Some grain boundaries appear as bright lines, due to strong surface topography (surface ledges), while other boundaries have much less topography. Enlarged regions have unit cells overlaid that illustrate relationships between the potential slip systems and slip traces. Slip planes are shown in shaded gray while the dashed lines represent the trace of the slip plane on the surface. The blue vectors on these planes show the $\langle 111\rangle$ slip directions. Each unit cell is designated with a Schmid factor (based on uniax-

\footnotetext{
${ }^{2}$ Local lattice curvature can also be quantified with other metrics such as the grain reference orientation deviation or kernel average misorientation.
}

ial tension) followed by a subscript indicating its ranking from high towards lower values of all of the possible 24 Schmid factor values. Pairs of unit cells across the grain boundaries are linked with arrows and the $m^{\prime}$ value for this pair of slip systems. This enables interpretation of features observed on the surface.

The $m^{\prime}$ values for each of the three grain boundaries described in Fig. 3 were evaluated using the strategy illustrated in Table 1. The seven highest Schmid factors among all potential slip systems (12 $\left.\begin{array}{llll}1 & 1 & 0\end{array}\right\}$ and $\left.12\left\{\begin{array}{lll}1 & 1 & 2\end{array}\right\}\right)$ are assembled in descending order in the first row for one grain and the first column for the other grain, i.e. the first row in Table 1 lists the seven highest Schmid factors for grain 55, and the first column similarly represents grain 82 . Schmid factor values in gold correspond to $\left\{\begin{array}{lll}1 & 1 & 0\end{array}\right\}$ slip planes and purple to $\left\{\begin{array}{lll}1 & 1 & 2\end{array}\right\}$ planes. The upper left part of Table 1 collects the slip system interactions that, because each pair combines large Schmid factor values, are most likely at the grain boundary. Each cell of the table provides the $m^{\prime}$ value associated with the geometry of the two slip systems, and $m^{\prime}$ values that are larger than 0.6 are typeset in bold. The slip system activity and slip transparency are combined in a parameter $m_{3 m}^{\prime}$ that isolates the most highly active slip systems. The $m^{\prime}$ values for slip system pairs with both Schmid factors greater than 0.35 are considered, and then the highest $m^{\prime}$ values (up to 3) associated with slip system pairs having the highest average Schmid factors are averaged (e.g. $m_{3 m}^{\prime}=$ average of the three underlined values in Table 1). A range of seven Schmid factors is considered, because the local stress tensor can vary significantly from uniaxial tension, which would alter the Schmid factors for each slip system, and therefore alter the relative order of likely slip system activity. Furthermore, it is known from the orientation maps in Fig. 2 that the grain orientations adjacent to the boundary differ from the average grain orientation, which also introduces uncertainty into above evaluation. Nevertheless, this is one of many ways to identify relevant values of $m^{\prime}$; this approach is based upon the assumption that no more than three slip system pairs in a given grain are likely to be dominant and involved in slip transfer.

The three example boundaries in Fig. 3 illustrate some scenarios that affect the calculation of $m_{3 m}^{\prime}$. In the grain boundary between grains 83 and 123, there is clear evidence of slip transfer across the boundary. In this case, the $m_{3 m}^{\prime}$ parameter is very high (0.97), so the appearance of slip traces that are linked across the boundary is not surprising. Within the grain, however, the lack of perfect alignment between the observed and computed slip traces suggests that a combination of slip on $\left\{\begin{array}{lll}1 & 1 & 0\end{array}\right\}$ and $\left\{\begin{array}{lll}1 & 1 & 2\end{array}\right\}$ planes with the same Burgers vector led to traces that are between those expected for either slip system. In the corresponding boundary in the LAM map in Fig. 2(a), there is little GND content on either side, except for grain 83 near the lower right triple point, where there is also evidence of a different active slip systems in the SEM image, along with increasing surface displacement (surface ledge) between the two grains near the triple point. This suggests that different slip systems with lower $m_{3 m}^{\prime}$ parameters were active at the triple point.

The accumulation of GND from poorer slip transfer conditions is examined in more depth using the boundary where 

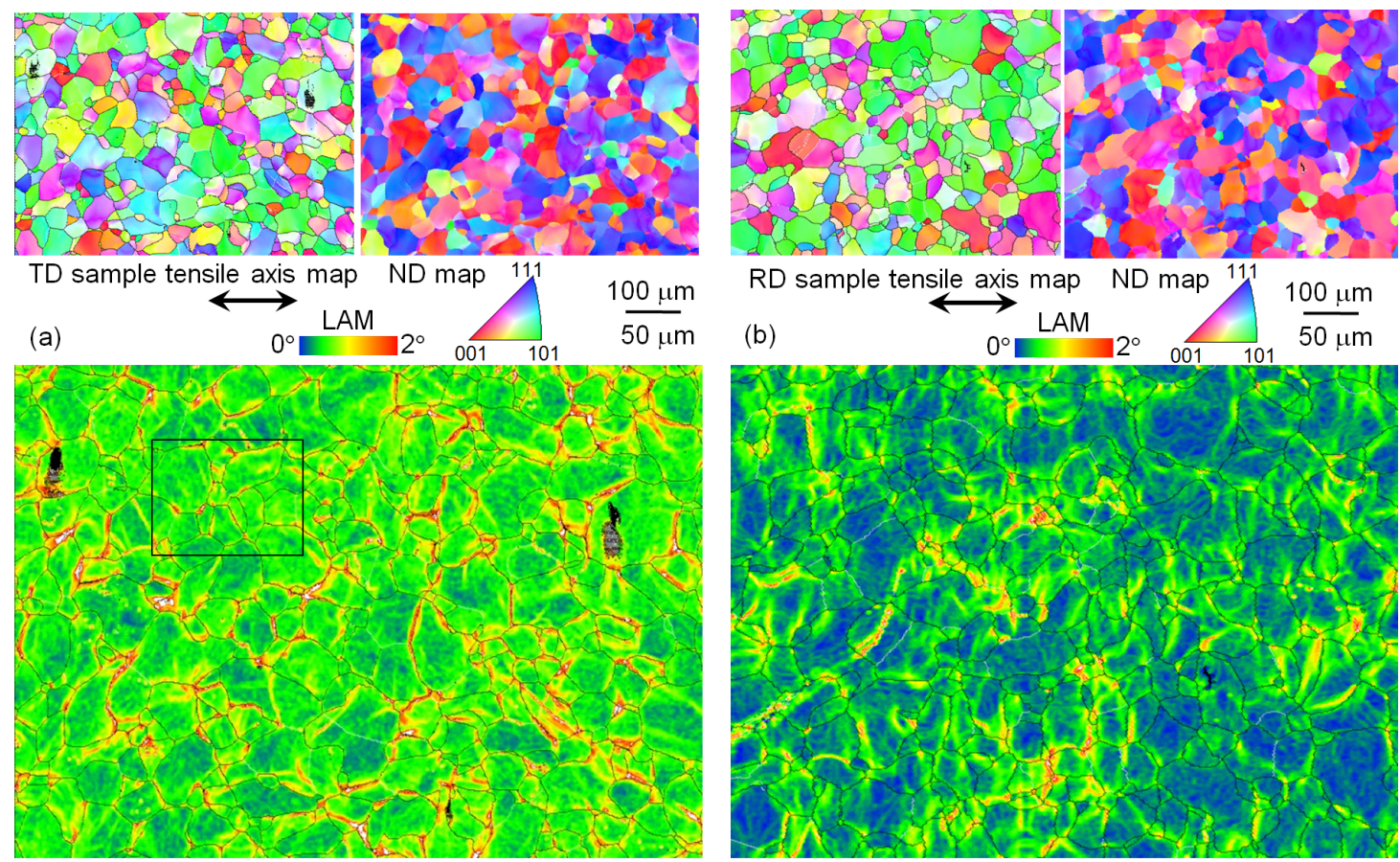

Figure 2: Microstructure patches on bent tantalum samples with the tensile axis horizontal at similar strains. The tensile axis and normal direction orientation maps for samples bent in the TD (a) and RD (b) are half-size, and the local average misorientation (LAM) maps are beneath, showing that the accumulation of geometrically necessary dislocations is different with deformation in the TD and RD directions. The inset identifies the region in Fig. 3.

there is a distinct surface ledge between grains 55 and 82 in the upper part of Fig. 3 and Table 1. Considering the $m^{\prime}$ values in the SEM image for slip systems pairs from left to right, the slip systems with very high Schmid factors and correspondingly obvious slip traces within the grains have a low $m^{\prime}$ value of 0.33 . There is a higher $m^{\prime}$ value of 0.74 , but this is for the $7^{\text {th }}$ most highly stressed slip system in grain 82 . There are three $m^{\prime}$ values that are above 0.9 between two similar slip systems on either side, but these slip systems have Schmid factors that are $4^{\text {th }}$ highest or lower, and there is no evidence for their operation. The last pair shows a rather low $m^{\prime}$ value of 0.67 for more highly stressed slip systems, and the slip trace matches well with observed traces in the lower half of grain 82 , but this slip system showed no obvious activity near the grain boundary. Given that the more favored slip systems did not have high $m^{\prime}$ parameters, the grain boundary represents a place of strain incompatibility, and the accumulation of GND content in the LAM map is consistent with the low $m^{\prime}$ parameters for active slip systems. Furthermore, the sense of shear in the two grains associated with highly stressed slip systems would create displacements with opposite directions at the surface for the observed slip systems. Specifically, the $2^{\text {nd }}$ highest system with obvious slip traces in grain 82 would move mass outward and to the left, while the most highly stressed system in grain 55 would move mass inward and to the left-so the formation of a surface ledge is not hard to imagine. Hence, formation of a surface ledge may be an indicator of a boundary with poor slip transfer conditions.

The third example in Fig. 3, the boundary between grains
126 and 136, also involves a surface ledge, but in this case, the $m^{\prime}$ values are much higher than in the prior example. This is more difficult to understand, but the angle $\theta$ of slip plane intersection in the grain boundary plane may be important here. From inspection of the surface ledge topography, the grain boundary plane is oriented similarly to the most favored slip systems (lower two prisms) in either grain. While the $m^{\prime}$ value is high, the angle $\theta$ between the two plane traces on the boundary plane may be rather large, given that the angle for two similarly oriented slip planes is minimized when the grain boundary is nearly perpendicular to the slip planes on either side, as depicted in Fig. 1. Indeed, there is apparent GND accumulation in this boundary visible in the LAM map, despite the high $m^{\prime}$ values. This suggests that all three angles defined in Fig. 1 may be important in predicting conditions for slip transfer. In order to fully assess the role of $\theta$, however, it is necessary to determine the grain boundary inclination experimentally.

An assessment of how well the LAM and associated GND content correlates with conditions favoring slip transfer is presented in Fig. 4, where the top two maps show the $m_{3 m}^{\prime}$ values on each boundary. Though the two samples were strained in different directions relative to the texture, there are no obvious differences between the maps for the TD (left) and the RD (right). The relationship between the $m_{3 m}^{\prime}$ values and the GND content (via the associated LAM value) was assessed by correlating the maximum LAM (if there was an strong peak) or dominant value (if there was no obvious peak) with the $m_{3 m}^{\prime}$ parameter using $5 \mathrm{LAM}$ value bins with $0.5^{\circ}$ increments and $10 \mathrm{~m}_{3 m}^{\prime}$ bins with 0.04 increments (> 500 boundaries were as- 

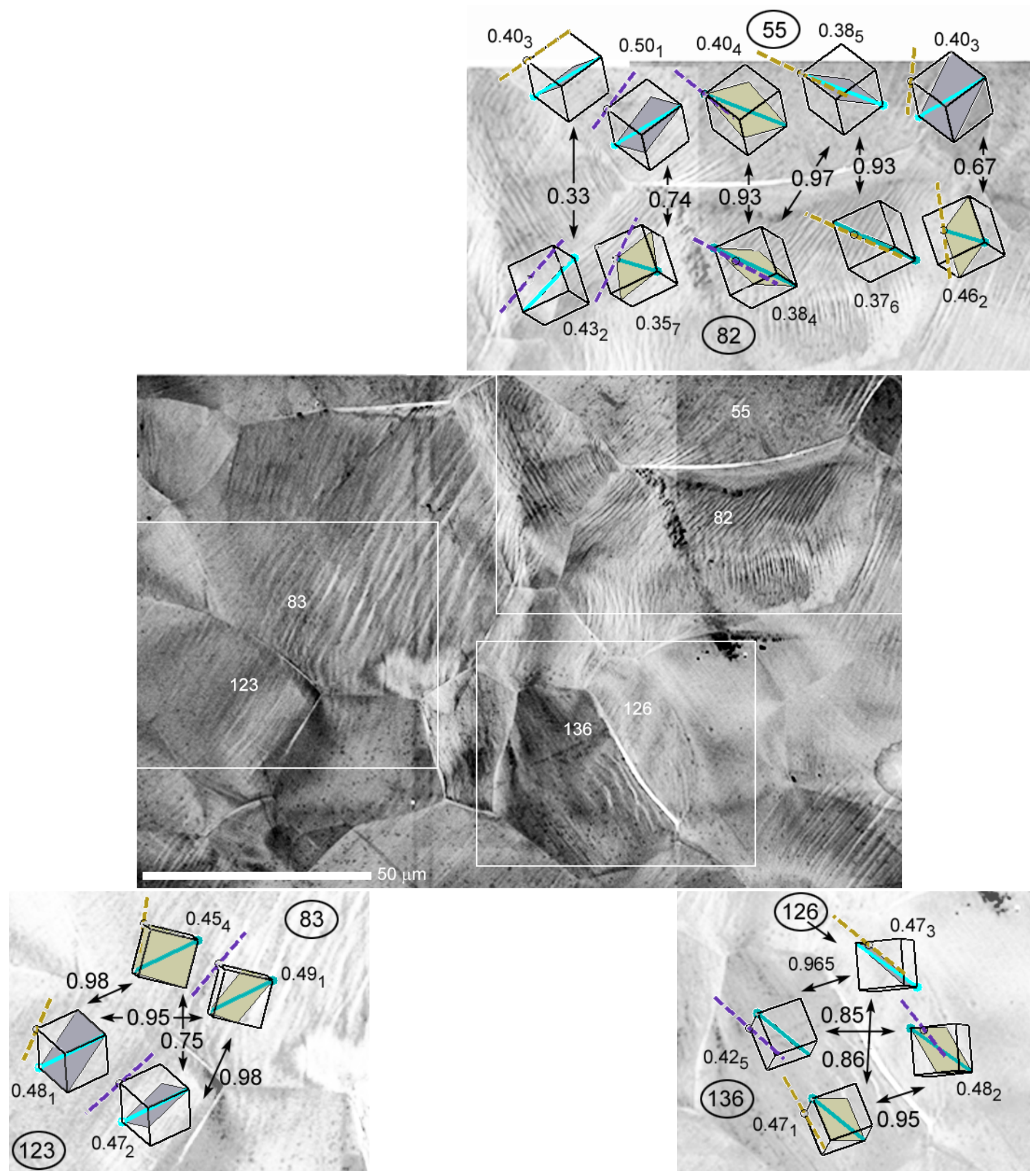

Figure 3: Secondary electron SEM image of a region of deformed Ta deformed in the transverse direction. At three particular boundaries, prisms illustrate potentially active slip systems, and their relationship with slip systems in the neighboring grains. Between the prisms, the $m^{\prime}$ parameter is noted, and the Schmid factor is noted on the other side of the prisms; the subscript indicates the rank in a sorted list of Schmid factor values. 
sessed). High LAM angles (red) represent high GND content, and low angles (blue-green) represent low GND content, and presumably, easier slip transfer conditions. For the TD sample, the distribution for boundaries with high GND content adjacent to grain boundaries is correlated with lower $m_{3 m}^{\prime}$ values. Similarly, boundaries with low GND content are correlated with higher $m_{3 m}^{\prime}$ values, and there is a smooth gradation between these two conditions. In the RD sample, the relationship between $m_{3 m}^{\prime}$ and GND accumulation is much less distinct; it is similarly present at high $m_{3 m}^{\prime}$ values, but it is inverted at lower values. Also, the regions with the highest $m_{3 m}^{\prime}$ values tend to be in grain interiors along low-angle boundaries, and not along high-angle grain boundaries. At present, the reason for this difference is not known, but the assumption of uniaxial tension and use of the average grain orientations clearly add a noise factor to the present calculations. Clearly, there is opportunity to discover better algorithms that can inform a computational crystal plasticity simulation of discrete grains about whether a grain boundary will resist dislocation slip transfer or not.

\subsection{TiAl}

TiAl has an fcc derivative crystal structure with alternating layers of $\mathrm{Ti}$ and $\mathrm{Al}$ along the $\langle 001\rangle$ direction. The regular fcc Burgers vector of $1 / 2\left[\begin{array}{llll}1 & 1 & 0\rangle\end{array}\right.$ is active on $\left\{\begin{array}{llll}1 & 1 & 1\end{array}\right\}$ planes but slip with a $\langle c\rangle$-component requires two 1/2[0 111$\rangle$ dislocations to maintain order, i.e., one $\left\langle\begin{array}{lll}0 & 1 & 1\end{array}\right]$ super-dislocation. Consequently, the four ordinary slip systems facilitate shape changes in the $\langle a\rangle-\langle b\rangle$ plane only; they are much easier to operate than the eight super-dislocation systems. To facilitate crystal deformation in the $\langle c\rangle$-axis direction, mechanical twins are activated, causing a relatively intense shear of 0.7 .

Slip transfer associated with mechanical twinning that leads to microcrack nucleation in TiAl has been examined in detail $[37,47,48]$. A fracture initiation parameter was developed that could distinguish the population of grain boundaries that developed microcracks from the population that did not. Many variations of this fracture initiation parameter were considered, as discussed in $[49,50]$, but

$$
F_{1}=m_{\mathrm{twin}}\left|\boldsymbol{b}_{\mathrm{twin}} \cdot \boldsymbol{t}\right| \sum_{\text {other }} \underbrace{\boldsymbol{b}_{\mathrm{twin}} \cdot \boldsymbol{b}_{\mathrm{other}}}_{\cos \kappa},
$$

which was the one initially published, seems to be the most robust version. This parameter combines three conditions that could be related to microcrack initiation due to twinning: (i) the Schmid factor $m_{\text {twin }}$ of an operating slip system, (ii) how the tensile traction $\boldsymbol{t}$ combined with the sense of shear $\left(\boldsymbol{b}_{\text {twin }}\right)$ favors a mode I crack opening, and (iii) how effectively twin shear can be accommodated by ordinary dislocations in the neighboring grain. The geometric considerations behind this parameter are illustrated schematically in Fig. 5.

This parameter is larger when the potential for slip transfer is large, i.e. when the twin shear can be well accommodated by slip in the neighboring grain. Maximal values of this parameter are reached if two or more slip systems imperfectly accommodate the twin shear since this results in a larger overall sum of $\cos \kappa$ terms than one well aligned slip system. This imperfect

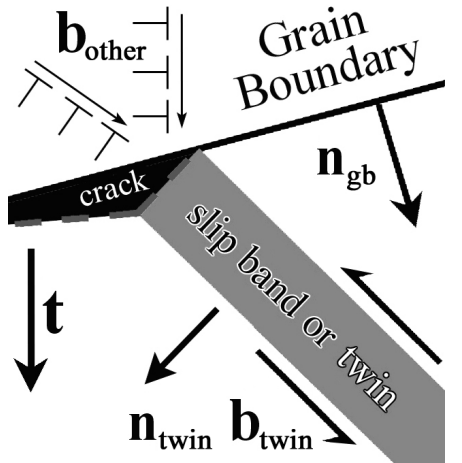

Figure 5: Stress state and geometric relationships between slip and twinning system across a grain boundary.

slip transfer maximizes residual dislocation content and energy within the grain boundary, which facilitates fracture.

One reason why $F_{1}$ worked well for TiAl, despite its disregard of slip plane alignment, might be related to the additional operation of $\left\{\begin{array}{llll}1 & 0 & 0\end{array}\right\}$ slip planes, which has been reported particularly in situations where dislocations interact with grain or twin boundaries [51]. Activity on $\{100\}$ planes doubles the number of available planes for slip transfer to occur on, which may lead to generally small — and thus indiscriminate-values of $\psi$ for any potential instance of slip transfer.

When evaluating $F_{1}$ in the boundaries that cracked, there was a high correlation between the twin system with the maximum Schmid factor and microcracking, because the higher Schmid factor led to more and larger twins, such that when they became sufficiently thick, cracks could be nucleated. Among the 11 cases examined in detail, there was only one outlier where the twin system with the second highest Schmid factor was responsible for forming microcracks. This case was examined with a crystal plasticity finite element simulation to examine the local stress state in the grain neighborhood, as discussed in [48]. This analysis showed that the local stress state at the fractured boundary led to a higher amount of shear on the twin system that generated the cracks, because the local stress state differed significantly from the global stress state. Thus, this analysis provided evidence that when the stress state was correctly assessed, the $F_{1}$ rule worked in every case.

\subsection{Slip transfer in hexagonal metals}

Because a fracture initiation parameter was effectively identified for TiAl, a material of low ductility, it is important to determine whether this idea is transferable to metals of higher ductility. Hexagonal metals are advantageous for this kind of research because there are fewer slip systems than in cubic metals, which makes experimental assessment easier. Also, understanding the origins of cracks is critically important in many applications [52-54]. Because planar slip is often observed in $\mathrm{Ti}$ and $\mathrm{Ti}$ alloys, the development of slip traces on previously polished surfaces clearly shows that some grains exhibit slip transfer, and others do not, as indicated in Fig. 6. The SEM image shows clear slip transfer between grains 3 and 9 , and 1 and 22 , and the annotated orientation map (b) shows that the 

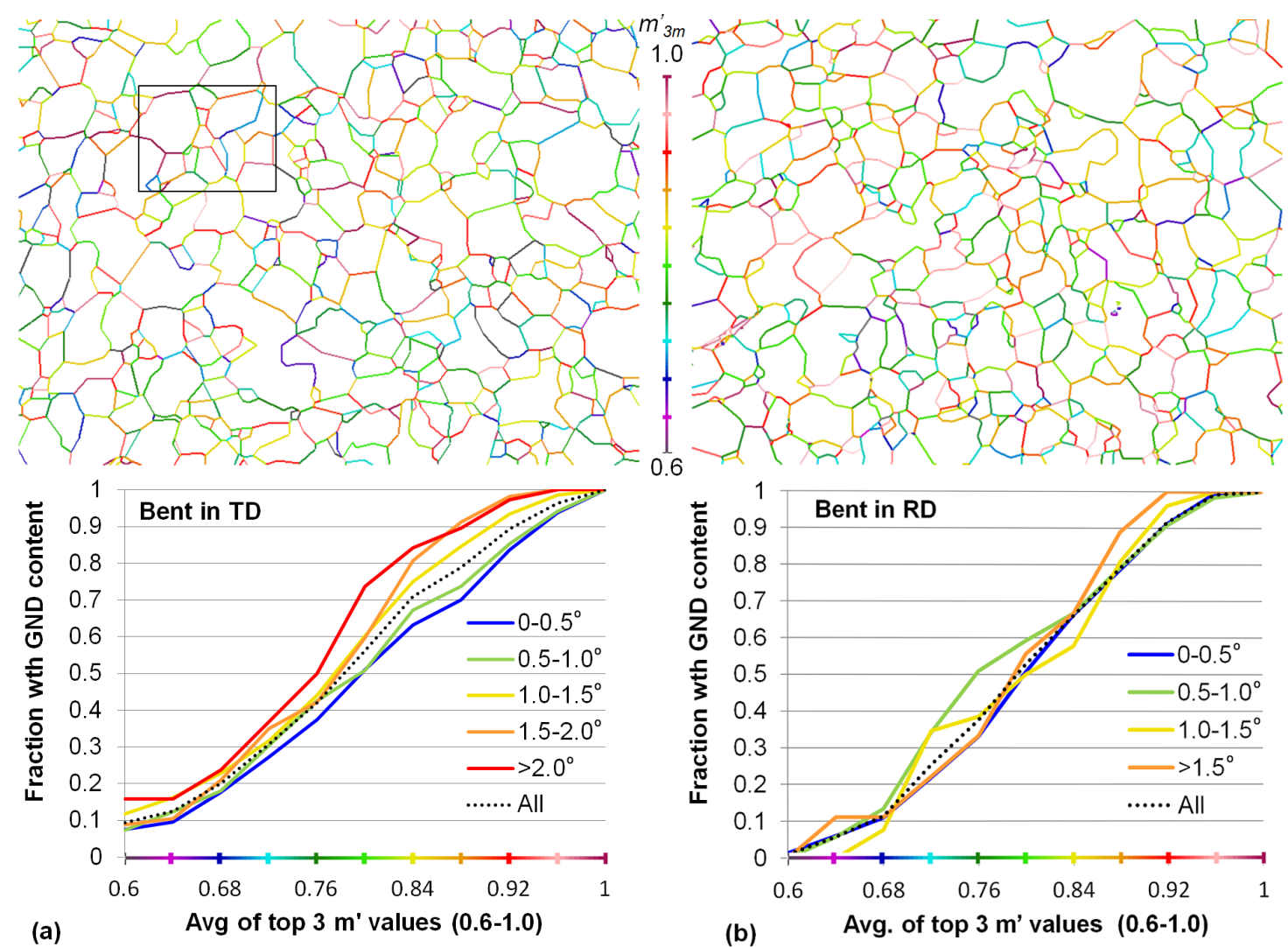

Figure 4: The $m_{3 m}^{\prime}$ parameter is plotted on the reconstructed boundary map. The cumulative fraction of boundaries with different local average misorientation (LAM) values are plotted against the $m_{3 m}^{\prime}$ parameter. The horizontal axis is color coded to the plotted $m_{3 m}^{\prime}$ values. The inset identifies the region in Fig. 3. 
$m^{\prime}$ parameter is greater than 0.9. Also, between grains 1 and 2, there is clear indication of slip to twin transfer, with a high $m^{\prime}$ value of 0.93 .

It is noteworthy that instances of slip transfer match up rather well at the grain boundary, yet the slip planes are not parallel. This is not easily explained, but there is the possibility that the free surface facilitates nucleation of slip in adjacent grains due to the lack of constraint, and slip bands develop from this nucleation process. There is faint evidence for slip transfer elsewhere, such as between grains 10 and 12, for basal slip. There are high $m^{\prime}$ values at many boundaries that involve pyramidal slip, such as between grains 9 and 10, in which there is questionable evidence of slip transfer; in this boundary, there is a noticeable presence of lattice curvature in the color gradient near the triple point, suggesting that no slip transfer took place.

Slip transfer by prism to prism slip is readily observed in pure $\mathrm{Ti}$ as illustrated in Fig. 6, but with alloying, the relative activity of basal slip increases [41]. Figure 7 illustrates a region of deformed microstructure after about 0.04 strain, where there is evidence for slip transfer with basal slip in the left and upper parts of the figure, where the blue slip traces are nearly parallel across several boundaries. In addition, there are some unusual surface ledges that developed in the interior of the central grain (similar features were observed in several other grains in the nearby microstructure). These surface ledges do not follow the traces of commonly observed slip systems, and they are not as straight as slip traces usually are. This provided motivation for simulating the deformation, to determine if modeling could provide insights.

This microstructural patch has been modeled using crystal plasticity finite element simulations, using two microstructure geometries, including a columnar microstructure based on an OIM map. Clearly such a columnar geometry is not realistic, but it is convenient, and simulations provide rough agreement with experimental observations (see [39]). Recently, this microstructure was characterized non-destructively in three dimensions using differential aperture X-ray microscopy (DAXM), which provides crystal orientation information at about $1 \mu \mathrm{m}$ spatial resolution up to depths of about 100$200 \mu \mathrm{m}$ [55]. From 'slices' of orientation map information, an estimate of the actual 3D geometry was determined, including orientations and shapes of subsurface grains illustrated in Fig. 8. Comparisons between the columnar microstructure model and the more accurate 3D microstructure against experimentally determined surface orientation maps before and after deformation show that the 3D microstructure makes a large improvement in simulating the details, particularly near grain boundaries, where the columnar model show the largest deviations from measurement. Thus, the correct grain geometry enables very good simulation of experimental observations.

As the computational model provides local stress states (tensors), they can be analyzed to identify conditions that lead to heterogeneous strains and to identify which slip systems are favored in different regions of a given grain. To examine what could account for the intragranular surface ledge development in the central grain interior, generalized Schmid factors at the boundary locations under the surface grain were investigated,
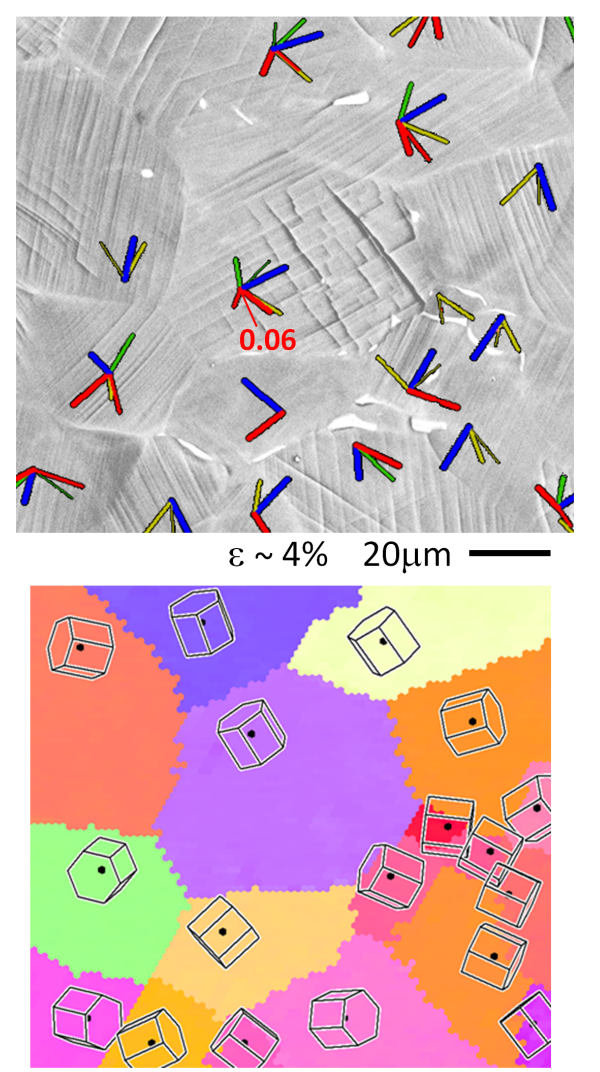

Figure 7: Surface topography of a tensile specimen after a strain of about 0.04 on a Ti-5Al-2.5Sn alloy, with traces of slip systems with high Schmid factor shown (blue: basal, red: prism, green: pyramidal $\langle a\rangle$, and gold: pyramidal $\langle c+a\rangle$ ); traces of surface ledges in the central grain do not agree with common slip traces. The orientation map shows the corresponding crystal orientations. 

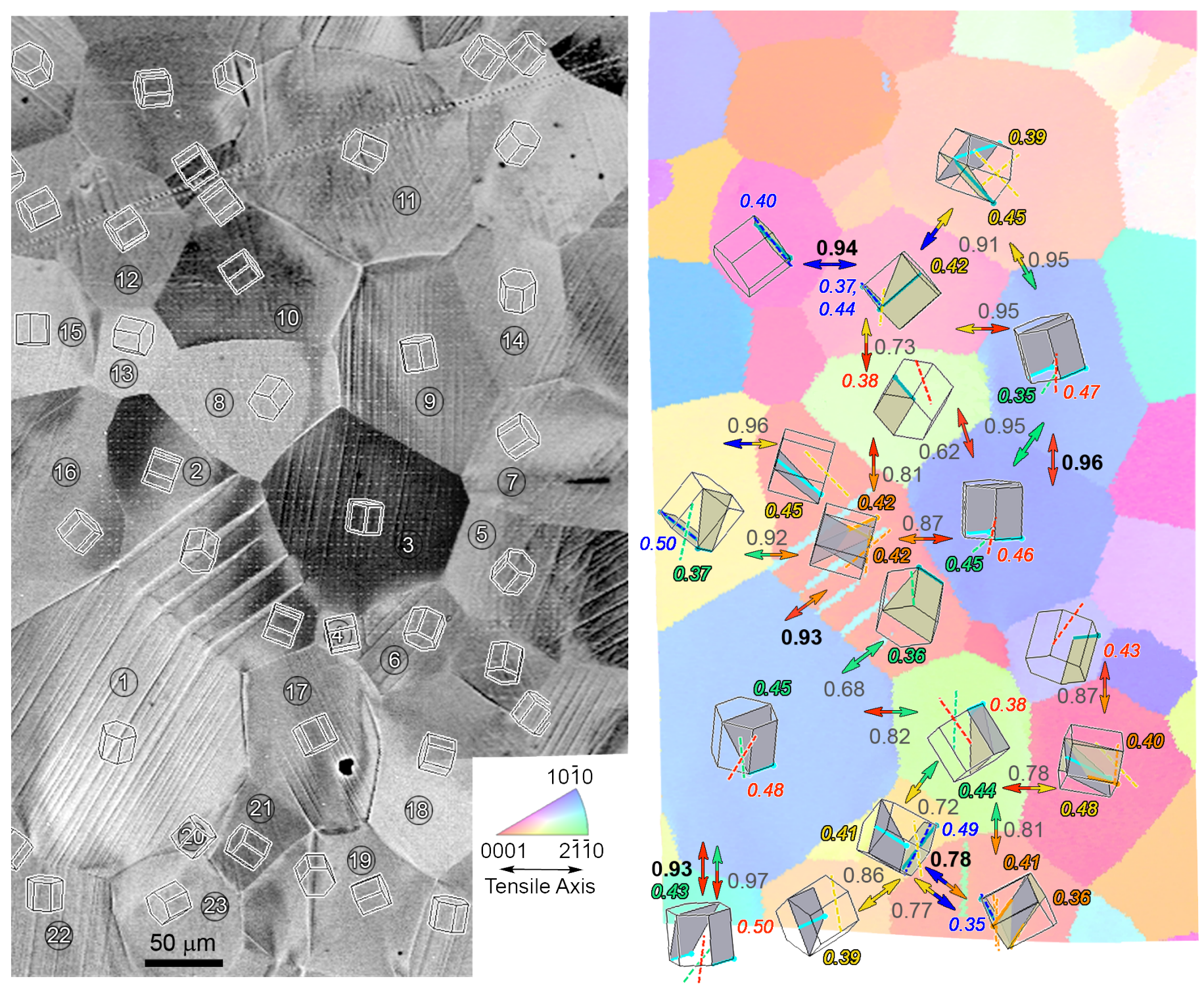

Figure 6: Examples of slip to slip and slip to twin transfer in CP Ti sample (a) SEM image of a patch of microstructure with about 0.015 strain where prism slip transfer is evident between grains 3 and 9, 22 and 1. Slip to twin transfer is clearly shown between grains 1 and 2. (b) EBSD inverse pole figure map showing computed $m^{\prime}$ parameters at most of the boundaries for specified slip systems; arrows link slip trace predictions from visualization of slip systems. red = prism, blue $=$ basal, green $=$ pyramidal $\langle a\rangle$ slip, gold = pyramidal $\langle c+a\rangle$ slip, and orange = tension twinning (T1). Observed evidence of slip transfer have $m^{\prime}$ parameters in black, the others are gray. 


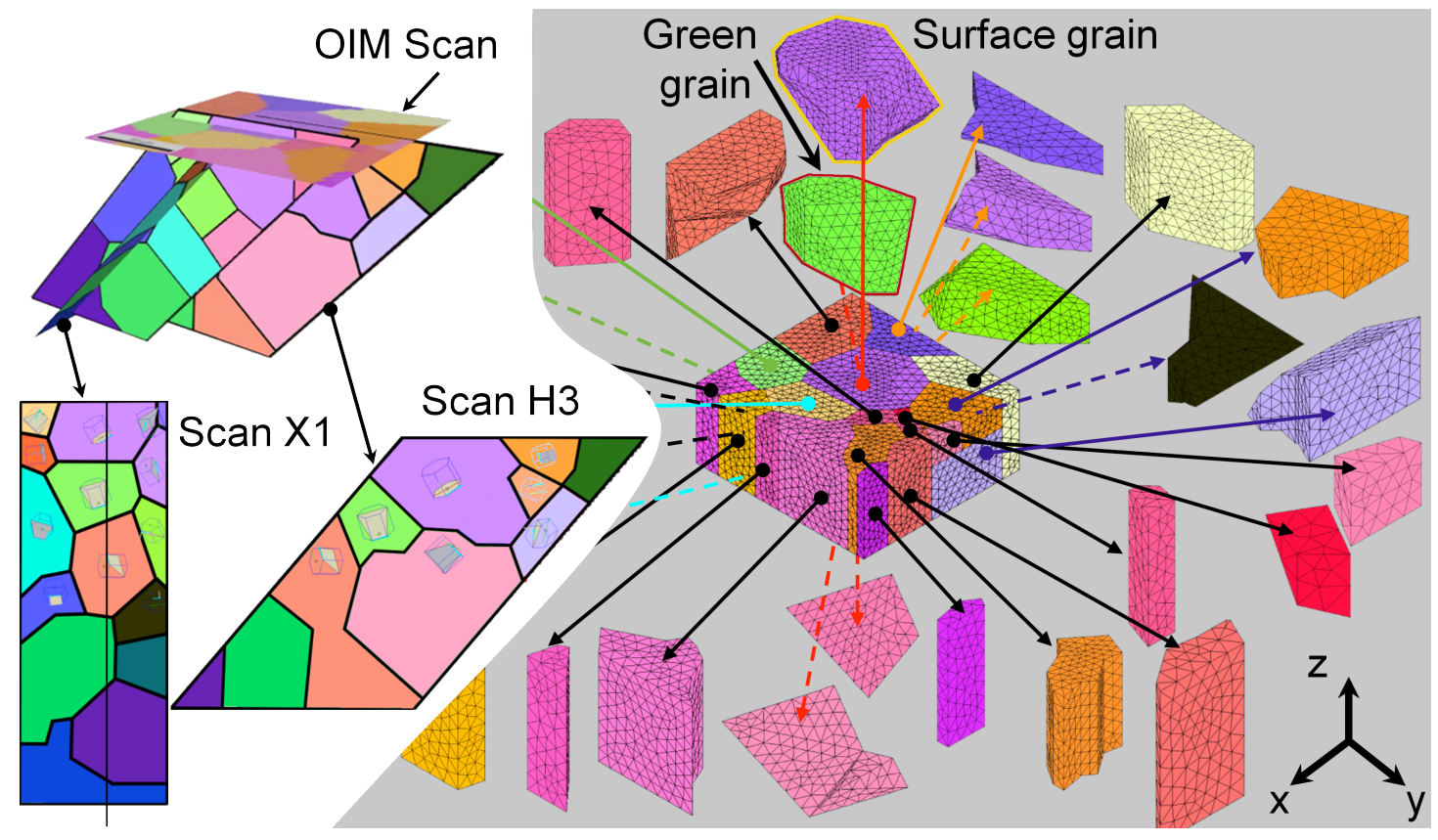

Figure 8: Orientation maps from DAXM slices allow construction of an estimated 3D microstructure. See text for discussion of outlined central purple and underlying green grains.

as shown in Fig. 9. ${ }^{3}$ In particular, the green-colored grain beneath the surface central grain Fig. 8 has a high Schmid factor for prism slip. This prism slip system has a high $m^{\prime}$ parameter of 0.93 with a second order prism slip system in the purple surface grain (outlined in gold), and the ledges in the surface grain line up closely with the slip trace of this system. At the interface with the green grain beneath (outlined in red), the Schmid factor for second order prism slip is low, but it is larger on the surface (also true for the visibly active basal slip). These different Schmid factor values result from a significant stress gradient from the surface to the boundary with the green grain, as illustrated in Fig. 10. The stress state in the surface grain is complex, close to a plane stress compression state on the surface, and becoming more triaxial toward the interface with the green grain. In contrast, the stress in the green grain does not vary much and is close to uniaxial tension. This analysis suggests that slip transfer from the underlying grain into a non-favored slip system can force its activation and generate unusual (and unexpected) surface features.

With a credible model for a patch of microstructure, it is possible to examine slip transfer parameter ideas with knowledge of the local stress tensor and the accumulated shear on each slip system. To this end, the accumulated shear was used to weight the $m^{\prime}$ parameter, and then summed to make a shearinformed version of a slip transfer parameter:

$$
m_{\gamma}^{\prime}=\sum_{\alpha} \sum_{\beta} \underbrace{m_{\alpha \beta}^{\prime}}_{\cos \kappa \cos \psi}\left(\gamma^{\alpha} \gamma^{\beta}\right) / \sum_{\alpha} \sum_{\beta}\left(\gamma^{\alpha} \gamma^{\beta}\right) .
$$

${ }^{3}$ The Schmid factor is formally defined only for uniaxial tension. A generalized Schmid factor, which describes the shear stress on a given slip system, can be computed from any stress tensor based on the Frobenius norm of the tensor.

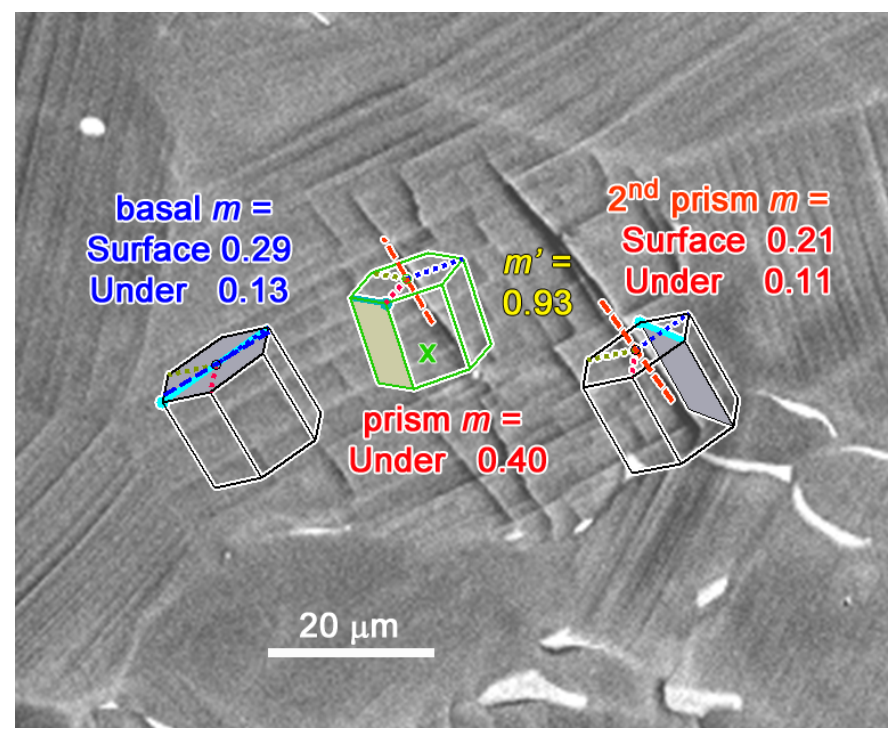

Figure 9: The green-colored subsurface grain illustrated at the top of Fig. 8 is oriented such that highly activated prism slip has a high $m^{\prime}$ value with second order prism slip system in the surface grain, which is consistent with the orientation and step geometry of the surface ledges. Arrows identify boundaries with evident slip transfer. 


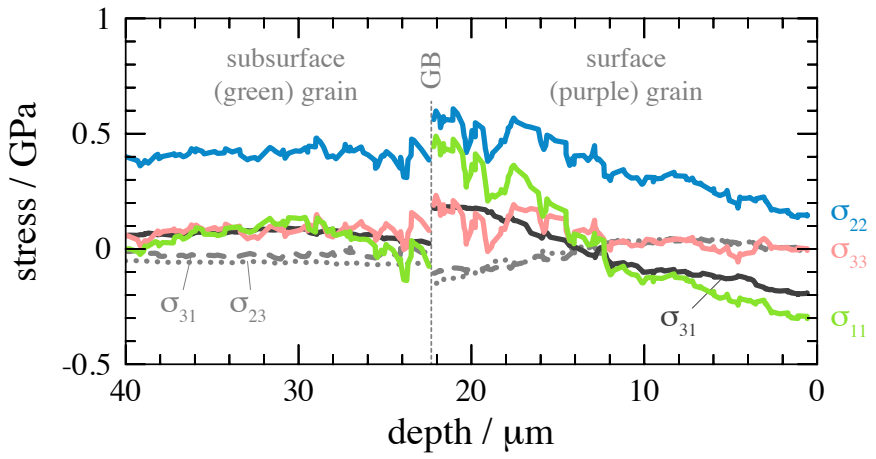

Figure 10: Components of the stress tensor evaluated at integration points within a cylindrical volume near the center of the surface grain (purple, see Fig. 8) as function of depth extending into the next (green) subsurface grain. In the surface grain the stress state gradually changes, while the subsurface grain experiences nearly uniaxial and largely constant tension along the '2' direction, consistent with the overall boundary condition.

The sum goes over all possible combinations of slip systems $\alpha$ in one, and $\beta$ in the other grain. This can be evaluated at every element pair along the grain boundary, so that variations in the amount of shear on each slip system will inform the local driving force for slip.

In like manner, the LRB parameter can be evaluated when the inclination of the grain boundary is known: ${ }^{4}$

$$
\operatorname{LRB}_{\gamma}=\sum_{\alpha} \sum_{\beta} \underbrace{\operatorname{LRB}_{\alpha \beta}}_{\cos \kappa \cos \theta}\left(\gamma^{\alpha} \gamma^{\beta}\right) / \sum_{\alpha} \sum_{\beta}\left(\gamma^{\alpha} \gamma^{\beta}\right) .
$$

There is also the possibility of combining the cosines of all three angles into a weighted sum:

$$
s_{\gamma}=\sum_{\alpha} \sum_{\beta} \underbrace{s_{\alpha \beta}}_{\cos \kappa \cos \psi \cos \theta}\left(\gamma^{\alpha} \gamma^{\beta}\right) / \sum_{\alpha} \sum_{\beta}\left(\gamma^{\alpha} \gamma^{\beta}\right) .
$$

Similarly, the $m^{\prime}$ parameter can be weighted using the Schmid factor $m$ on each slip system as a metric for the magnitude of slip transfer:

$$
m_{m}^{\prime}=\sum_{\alpha} \sum_{\beta} \underbrace{m_{\alpha \beta}^{\prime}}_{\cos \kappa \cos \psi}\left(m^{\alpha} m^{\beta}\right) / \sum_{\alpha} \sum_{\beta}\left(m^{\alpha} m^{\beta}\right) .
$$

All four parameters are compared in Fig. 11. The three slip transfer parameters weighted by accumulated shear (Fig. 11ac) give similar variations on the same boundaries, though the details vary. The boundaries with the highest slip transfer values are the lower left and upper boundaries of the central grain, where slip transfer is evident, arrowed in Fig. 9. Also, the boundaries colored in dark red in the lower right part of Fig. 11a-c have high slip transparency. It is difficult to even see these boundaries in Fig. 7, indicating a lack of disturbance to the slip bands at these boundaries.

\footnotetext{
${ }^{4} \mathrm{Here}$, the inclinations in the vicinity of the central grain are estimated from
} DAXM scans.
In contrast, the Schmid factor based $m_{m}^{\prime}$ varies over a small range (Fig. 11d), such that no variation is evident along the boundaries. Because $\dot{\gamma} \propto \tau^{n}$, where $n \approx 20$, the shear strain rate $\dot{\gamma}$ is amplified by the resolved shear stress $\tau$ raised to a large exponent, the accumulated shear strain is dominated by slip systems that have high Schmid factors. This amplification does not occur for the Schmid factor, as inactive slip systems have Schmid factors that only differ by a factor of 2 or 3 , not orders of magnitude. Hence, when the logarithmic scale used in Fig. 11d is changed to a narrow linear scale and replotted in Fig. 11e and $\mathrm{f}$, the pattern of low and high transparency boundaries is similar to that found using shear as a weighting factor (in Fig. 11ac), which shows that even though irrelevant Schmid factor values contribute much to the sum, boundaries with higher transparency can still be discerned. Use of the global Schmid factor in Fig. 11e rather than the local Schmid factor provides a result that is very similar to the local Schmid factor result shown in Fig. 11f. This suggests that the slip transfer character can be estimated from an experimental measurement that uses orientation information on either side of the grain boundary to assess the slip transfer parameter. Finally, the parameters identified in Eqs. (2) to (5) clearly contain much irrelevant information. Thus, strategies to meaningfully filter out the most relevant slip transfer components, such as the strategy used for $m_{3 m}^{\prime}$ in the tantalum sample discussed above, need to be explored.

\subsection{Twin transfer in hexagonal metals}

Twinning as a possible accommodation mechanism for heterogeneous strain in neighboring grains was investigated in commercially pure titanium with a moderately strong (about 6 times random) $\left\{\begin{array}{lllll}0 & 0 & 0 & 1\end{array}\right\}$ fiber texture [38, 56-58]. Four-point bending specimens were selected in two orientations such that the tensile surface strain was either introduced along the preferred $\left\langle 0 \begin{array}{lll}0 & 0 & 1\end{array}\right\rangle$ direction or at $45^{\circ}$ from it. In commercial pu-

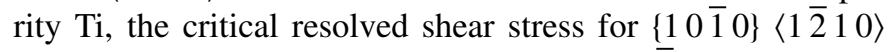

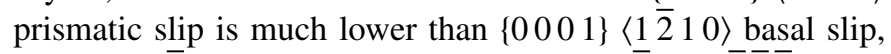

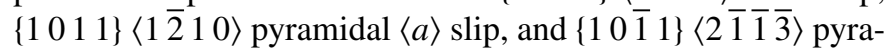
midal $\langle c+a\rangle$ slip [59-64]. Therefore, in the $\langle 00001\rangle$ sample orientation, most grains are in a 'hard' orientation, as their $\langle c\rangle$ axis is aligned close to the tensile direction. This alignment

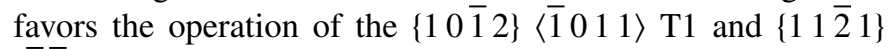
$\langle\overline{1} 126\rangle \mathrm{T} 2$ extension twins as well as pyramidal $\langle c+a\rangle$ slip due to comparably high resolved stresses on those systems (i.e. large Schmid factors). The second sample orientation had a mix of 'soft' and 'hard' grain orientations.

The majority of twins in Ti, $\left\{\begin{array}{llll}1 & 0 & \overline{1} & 2\end{array}\right\}\left\langle\overline{1} \begin{array}{llll}0 & 1 & 1\rangle\end{array}\right\rangle$ T extension twins, have a shear of 0.17 , which is small compared to the 0.70 twin shear strain in TiAl. Consequently, there is little evidence of damage at modest strains in Ti. However, extension $\left\{\begin{array}{lll}1 & 1 & \overline{2} \\ 1\end{array}\right\}$ $\langle\overline{1} \overline{1} 26\rangle \mathrm{T} 2$ twins in Ti have a shear strain of 0.67 . Though rare (observed in 6 of 1920 grains) microcracks have been observed in connection with T2 twins (Fig. 12) for probably the same reasons as in TiAl. Because the locations where T2 twins interact with grain boundaries have large local shears, it is difficult to extract information about accommodating slip operation, but twin accommodation is obvious, as illustrated in Fig. 12, and discussed in more depth in [58]. 


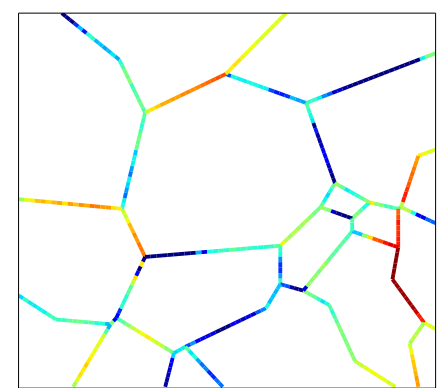

(a) $m_{\gamma}^{\prime}$

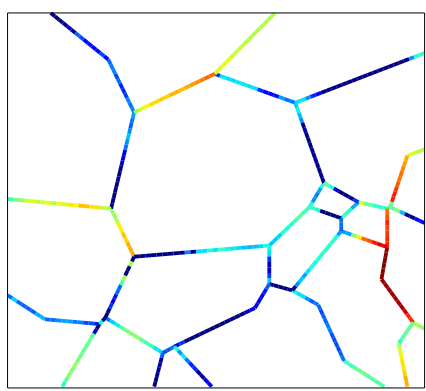

(c) $s_{\gamma}$

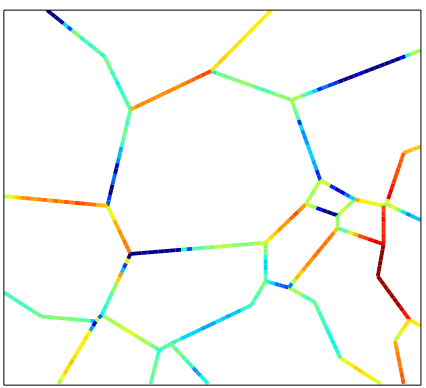

(b) $\mathrm{LRB}_{\gamma}$

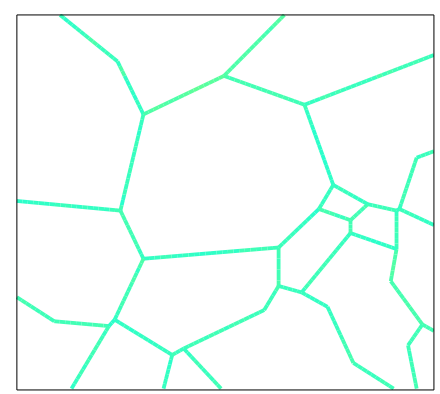

(d) $m_{m \text {-local }}^{\prime}$

0.1

$\uparrow$ logarithmic

0.25

$\downarrow$ linear

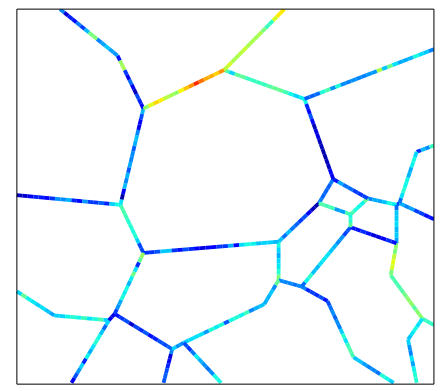

(e) $m_{m \text {-global }}^{\prime}$

(f) $m_{m \text {-local }}^{\prime}$
Figure 11: Maps of slip transfer parameters defined in Eqs. (2) to (5) show similar trends but differences in detail; a-d uses a logarithmic scale, while e and f uses a narrow linear scale.

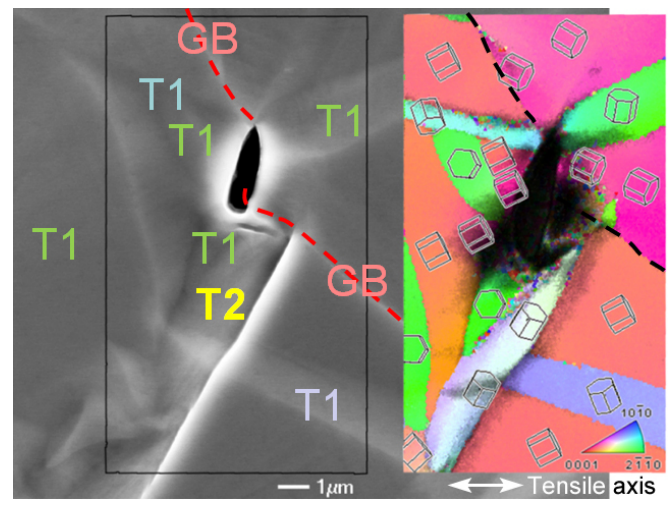

Figure 12: Microcrack nucleation at a $\left\{\begin{array}{lll}1 & 1 & \overline{2} \\ 1\end{array}\right\}\langle\overline{1} \overline{1} 26\rangle \mathrm{T} 2$ extension twin, with complex accommodation by $\left\{\begin{array}{ll}1 & 0 \\ 1 & 2\end{array}\right\}$ $\langle\overline{1} 011\rangle \mathrm{T} 1$ extension twins on either side of the boundary.

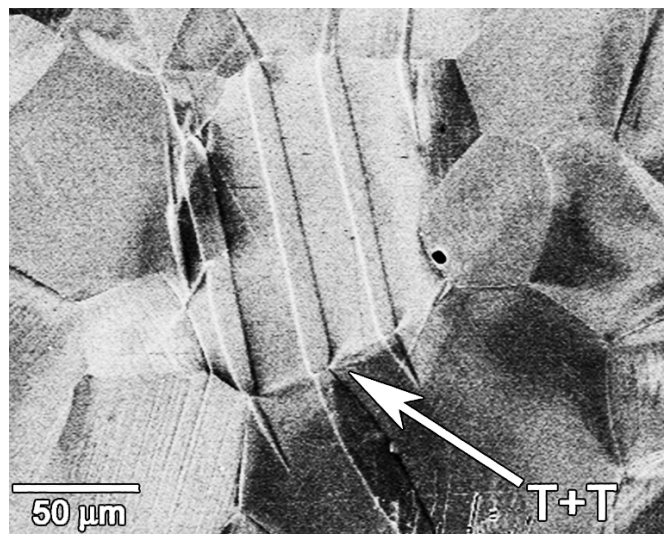

Figure 13: Example of slip transfer across grain boundaries by twinning ("T+T").

Out of all of the grains that showed evidence of T1 extension twinning, a fraction of about $1 / 3$ appeared to have twinning correlated to localized deformation in a neighboring grain, as exemplified in Fig. 6, where slip stimulated twins formed between grains 1 and 2, and in Fig. 13, where a twin in one grain led to formation of a twin in the neighboring grain [56]. Such effects are much more commonly observed in magnesium alloys [65-67]. These two cases where alignment of slip or twins in the initiating grain leads to formation of twins in the neighboring grain are analyzed in the following.

Soft grains with clear indications of operating prism slip (traces) located next to hard ${ }^{5}$ grains were categorized based on the presence of correlated $\mathrm{T} 1$ twin activity in the neighboring (receiving) hard grain. For such pairs, 15 cases showed no twin activity ("S+0") and in 11 cases correlated activity ("S+T") across the grain boundary was observed. If a twinning system was activated in the hard grain, it always had the highest $m^{\prime}$ value among the six possible T1 twinning systems, but was not necessarily the one with the highest Schmid factor based on global unidirectional tension. The corresponding distributions of (global) twinning Schmid factor and $m^{\prime}$ for both grain pair populations are shown in Fig. 14. While the Schmid factor distributions are very similar, the $m^{\prime}$ distributions clearly separate the two populations (there are two $\mathrm{S}+0$ outliers that might develop correlated twinning at higher strain). This suggests that nucleation of favorably oriented twins is triggered by altering the stress state close to the grain boundary due to localized (prismatic) slip.

Figure 15a presents the correlation between $\kappa$ and $\psi$, i.e. the alignment of displacement vectors and shear planes, for $\mathrm{S}+0$ and $\mathrm{S}+\mathrm{T}$ conditions. Cases where slip is correlated with twinning (open circles) cluster in the lower-left corner at high $m^{\prime}$ values in accord with the right-most distribution of Fig. 14. This plot examines whether there is a preference for slip transfer based on slip vector $\kappa$, or plane alignment $\psi$. There is no clear trend of either systematically lower $\kappa$ or $\psi$ observed when

\footnotetext{
${ }^{5}$ Soft grains are defined as having at least one prism slip system with $m>$ 0.4 , while hard grains have no prism system with $m>0.15$.
} 


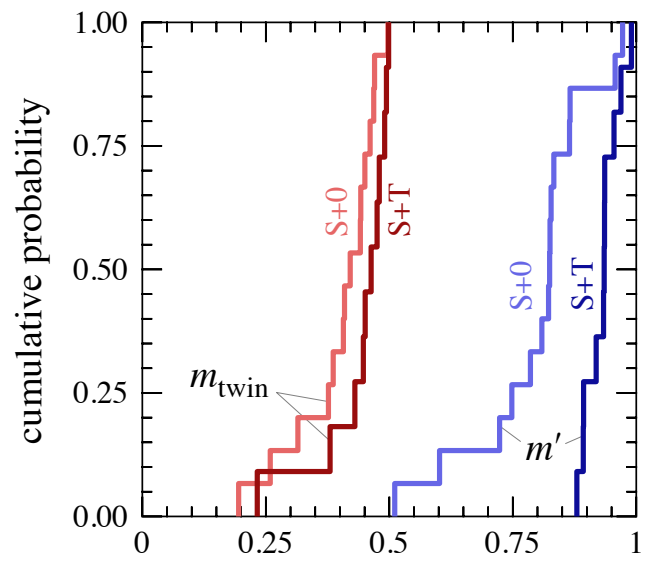

Figure 14: Distribution of twin Schmid factor in receiving hard grains (left, red) and deformation system alignment between soft and hard grains (blue, right) for cases with $(\mathrm{S}+\mathrm{T})$ and without $(\mathrm{S}+0)$ twin activity correlated to prismatic slip in soft neighboring grains.

slip is transmitted (circles), or when the most favorably oriented twinning system remains inactive (dots).

In the sample with predominantly hard orientations, an evaluation of similar crystallographic relationships was done for twinned grains that showed correlated twinning ("T+T") activity across an impinged boundary, e.g. Fig. 13. Figure $15 \mathrm{~b}$ presents the geometrical alignments found for correlated twin activity. Similar to the case of slip-correlated twinning, the vast majority of observations clusters at high $m^{\prime}$ values. However, this $m^{\prime}$ distribution has a more pronounced low-end tail with eight values (about $1 / 3$ of all cases) falling into the range of $0.8-0.1$. For all except one of those situations, the low $m^{\prime}$ is predominantly due to a relatively larger misalignment $\psi$ of the twin planes - the misalignment $\kappa$ of Burgers vectors typically stays below $30^{\circ}$. This larger tolerance of twinning plane misalignment suggests that twins in the receiving grain are able to adjust their spatial expansion in such a way as to follow the trace imposed by the twin in the originating grain. Such a close alignment of a twin triggered by another one has indeed been observed by Fernández et al. [68] who investigated twin-twin geometries in three dimensions using serial sectioning.

Every $\mathrm{T}+\mathrm{T}$ case was visually inspected to identify the likely order of twin generation based on the extent that the twins propagated in the neighboring grains (overall kinematic appearance). Figure 16 presents the distribution of Schmid factor ratios between the triggering and triggered twin system based on an assumed unidirectional state of tension at 16 grain boundaries for which the direction of twin propagation was obvious. This distribution exhibits a marked shift to values above unity, with a probability of only $2 \%$ that its median is unity. This shift is consistent with the view that the triggering twins grow because they are well aligned with respect to the global state of stress (e.g. arising from uniaxial tension). Once they impinge on a grain boundary, the resulting local alteration of the stress field in the neighboring grain ahead of the twin tip is able

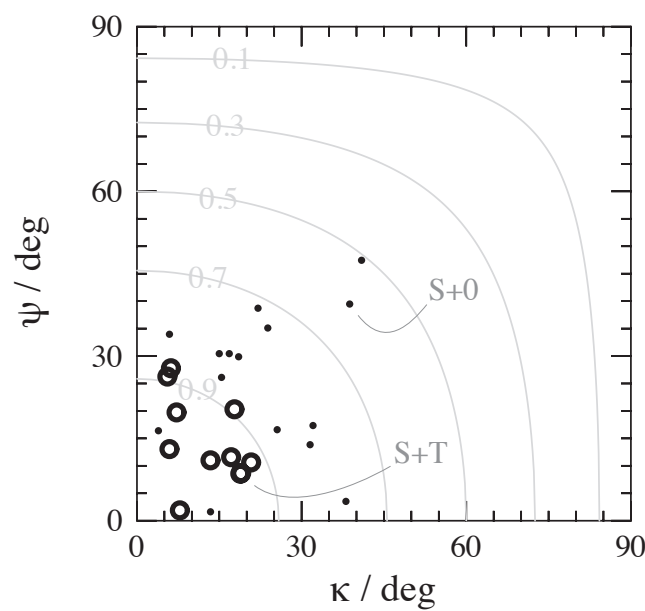

(a) Relationship between observed twin system (circles) or bestaligned but inactive twin system (dots) in a grain with localized slip activated in the neighboring grain. Activated twin systems cluster in the lower-left corner and have consistently larger $m^{\prime}$ values than those of the best-aligned inactive ones except for two (dots) of exceptionally high $m^{\prime}$.

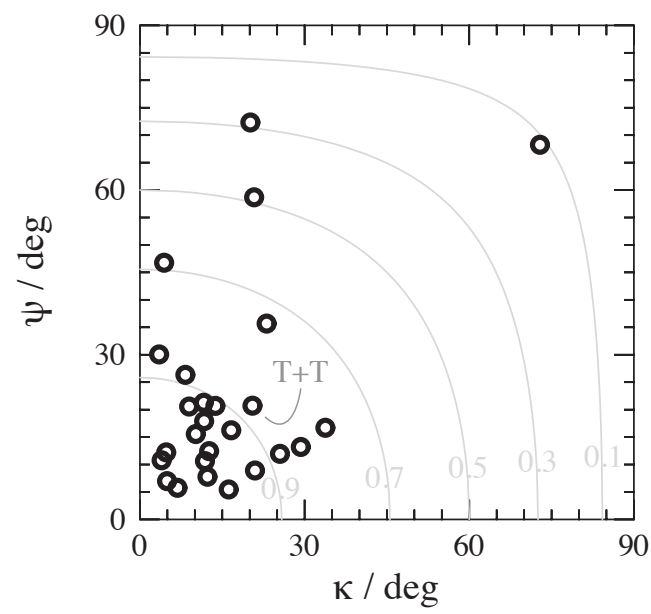

(b) Observed twin system (circles) in a grain next to one with twin activity. Small $\kappa$ is more frequently observed than small $\psi$. The majority of cases exhibits $m^{\prime}>0.8$.

Figure 15: Geometric correlation between observed (or bestmatching but inactive) twin system in a receiving grain and either slip traces a or twins $b$ that impinge on the grain boundary from a neighboring grain. Angles $\kappa$ and $\psi$ quantify misalignment between Burgers vectors and twinning/slip plane normals, respectively. Isolines of $m^{\prime}=\cos \kappa \cos \psi$ are plotted and labeled in light gray. There is a slightly greater tolerance for variation in slip plane alignment than slip direction alignment. 


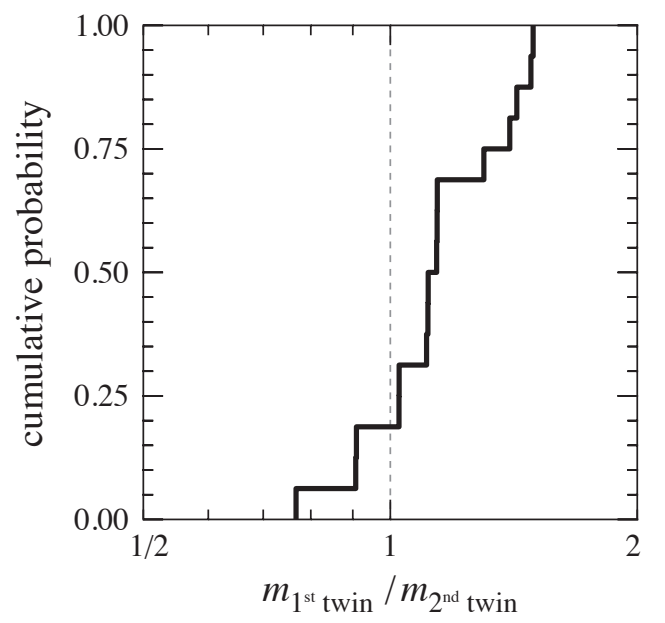

Figure 16: Distribution of Schmid factor ratio between originating $\left(1^{\text {st }}\right)$ and triggered $\left(2^{\text {nd }}\right)$ twin system. The observed shift to values larger than 1 suggests that the originating twin system is largely dictated by the global stress, while the triggered twin system responds to a (different) local stress state induced by the impinging twin.

to trigger activity on a twin system ${ }^{6}$ that is less favored by the global state of stress. Therefore, the (global) Schmid factor of a triggered twin system is usually lower than that of the impinging one, since otherwise the former would likely have acted as a trigger itself.

\subsection{Subsurface twin nucleation conditions}

Thus far, surface observations indicate that twin nucleation by accommodation of localized shear from a neighboring grain is an important grain boundary mediated deformation mechanism. As there is less mechanical constraint on the surface than in the interior, it is also important to determine if this process also occurs below the surface where there is more constraint. This is currently being assessed using 3D X-ray diffraction on in-situ deformed tensile samples with the same microstructure as shown in Fig. 6; initial results from this ongoing investigation are presented briefly. This non-destructive method relies on collecting diffraction patterns at small increments of strain from which both the elastic stress state and the emergence of new orientations (twins) can be identified as a function of strain [45]. An elaborate diffraction tomographic analysis is required to extract the stress state and spatial locations of grains with twins nucleated within them [69]. From this analysis, several twin nucleation events have been identified, which are described in Table 2. The first two columns identify the sample and layer of material interrogated along the tensile axis and the engineering strain where a twin nucleated and grew large enough to detect. The third column identifies the Schmid factor rank of the observed twin; the twin system with highest Schmid factor was

\footnotetext{
${ }^{6}$ The triggered system is likely well aligned with the triggering system according to $m^{\prime}$.
}

Table 2: Summary of subsurface twinning events.

\begin{tabular}{ccccccc}
\hline $\begin{array}{c}\text { Sample } \\
\text { layer }\end{array}$ & $\begin{array}{c}\text { Eng. } \\
\text { strain }\end{array}$ & $\begin{array}{c}\text { Twin Schmid } \\
\text { factor rank }\end{array}$ & & \multicolumn{2}{c}{$m^{\prime}$} & \multicolumn{2}{c}{$m$} \\
\cline { 1 - 1 } A-1 & $1.50 \%$ & Lowest & & 0.95 & $\operatorname{pyr}\langle a\rangle$ & 0.48 \\
A-2 & $1.50 \%$ & Highest & & & \\
A-3 & $1.50 \%$ & $2^{\text {nd }}$ lowest & & 0.96 & basal & 0.46 \\
B-1 & $1.13 \%$ & Highest & & 0.89 & pyr. $\langle c+a\rangle$ & 0.52 \\
B-2 & $1.56 \%$ & $2^{\text {nd }}$ lowest & & 0.96 & T1 twin & 0.50 \\
B-3 & $1.74 \%$ & Lowest & & & \\
B-3 & $1.74 \%$ & Lowest & & 0.95 & $2^{\text {nd }}$ pyr. $\langle c+a\rangle$ & 0.57 \\
\hline
\end{tabular}

activated in only two of seven observations. In five of the seven twins, the last two columns indicate whether the twin had a high $m^{\prime}$ value with a slip system in a neighboring grain, the slip system, and its Schmid factor based on the (average) stress tensor $^{7}$ operating on the neighboring grain within the same layer. ${ }^{8}$ This assessment identifies the possibility of an S+T event based upon the estimated geometry; the twin in layer A-3 is the most likely instance of $\mathrm{S}+\mathrm{T}$ twin nucleation due to basal slip being more likely to be strongly activated than the others. This is difficult to prove, as localized slip bands cannot be identified in Xray diffraction data, though it may be possible to extract some strain information from peak shape analysis based on methods described in [70]. This table shows that the nucleation conditions vary significantly from one event to the other, such that there is no obvious trend. Clearly the stress state and orientation of the neighboring grains have a large influence on twin nucleation, and the local stress states near the twin differ from the grain average stress state [71].

\subsection{Slip transfer across phase boundaries}

In the above sections, transfer of deformation activity has been considered between grains of the same phase. In most $\mathrm{Ti}$ alloys, in addition to the hexagonal $\alpha$ phase, the body-centered cubic $\beta$ phase is present to some extent. In the Ti-5Al-2.5Sn alloy, there are pockets of $\beta$ in grain boundaries and triple junctions, evident as white patches in Fig. 7. In a study of slip transfer between $\alpha$ and $\beta$ phases, a similar statistical characterization was done. However, only cases are considered for which surface slip traces were observed in both of two neighboring phases. If the slip traces are aligned at the phase boundary the situation were termed " $\mathrm{S}+\mathrm{S}$ ", while for non-correlated traces were termed "S+0". The results in Fig. 17 show more datum points closer to the horizontal axis than the vertical axis, suggesting that the slip plane alignment may account for more of the observed slip transfer than alignment of the Burgers vectors [72]. The correlation is not as convincing as with the $\alpha$ to $\alpha$ grain boundary analysis, as there are observations with large

\footnotetext{
${ }^{7}$ Generalized Schmid factors are greater than 0.5 in some instances because the grain average stress tensor differs significantly from uniaxial tension.

${ }^{8}$ This analysis does not yet include $m^{\prime}$ analysis for grains that are above and below the parent grain, which will be reported in the future.
} 


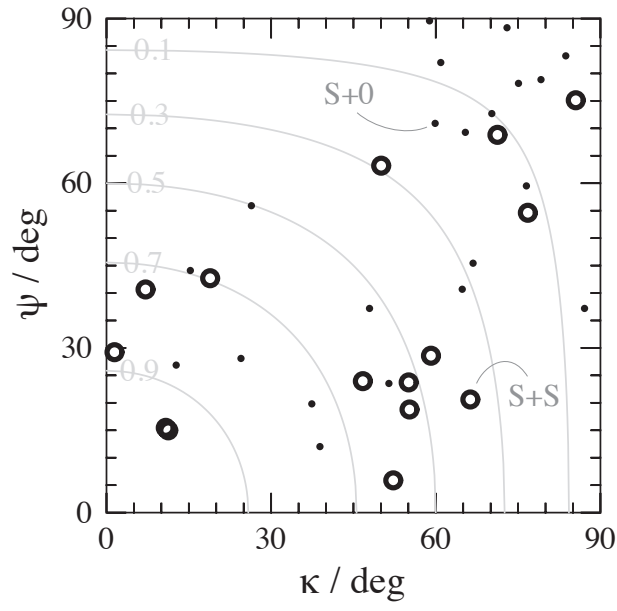

Figure 17: Geometric correlation between two observed slip systems in neighboring grains that have traces aligned ("S+S") or not aligned ("S+0") at the $\alpha / \beta$ phase boundary. Angles $\kappa$ and $\psi$ quantify misalignment between Burgers vectors and slip plane normals, respectively. Isolines of $m^{\prime}=\cos \kappa \cos \psi$ are plotted and labeled in light gray. The $\mathrm{S}+\mathrm{S}$ data being systematically closer to the horizontal than the vertical axis suggests that the alignment of plane normals is of greater importance for slip transfer than the slip direction alignment.

misorientations (upper right part of the plot). Also, this differs from observations in TiAl where the slip plane did not matter much, and with slip transfer in the $\alpha$ phase in titanium where the alignment of the Burgers vectors was a stronger indicator of slip transfer than that of plane normals.

\section{Discussion}

Consistent with [26], the results presented here illustrate how the geometry of the slip systems at the grain boundary needs to be integrated with their activity to determine the nature of slip transfer process. A number of different approaches to account for the geometry and activity of slip systems and their roles in strain transfer have been assessed. Different alloy systems appear to follow different dependencies on these variables. In several cases, the slip direction is more influential on slip transfer than the slip plane, such as in TiAl, where the fracture initiation parameter does not even consider the slip planes. In other cases, such as in titanium alloys, both the slip plane and direction, described by $m^{\prime}$, must be included. The importance of the angle of intersection of the slip plane traces on the grain boundary plane, $\cos \theta$, remains an open question. The strong dependence of grain boundary resistance on the activated slip systems and the geometric relationships between them and the grain boundary is exemplified by the two tantalum samples that share the same microstructure but show quite different slip transfer characteristics when deformed in different directions. All of these studies show that it is important to identify which slip systems are active in the context of slip transfer.
Comparison of crystal plasticity finite element simulations of quasi-3D (columnar grain model) and true 3D microstructures with experimental characterization reveal the need to have a good representation of the 3D microstructure in order to analyze the local deformation behavior involved in strain transfer at grain boundaries. Accurate simulations allow evolving local stress tensors to be estimated, and consequently the spatial variability of the driving force for activating a particular slip system can be identified. For example, operation of an unexpected slip system can be stimulated by a slip transfer condition that provides a high local driving force for its activation. Simulations of real microstructures are particularly useful for evaluating results from experimentally characterized microstructures.

It is also likely that deformation behavior on the surface, as examined experimentally in most of the studies presented here, differs from the highly constrained conditions in the bulk of the material. The fact that slip band features on both sides of grain boundaries are commonly correlated (despite the fact that the planes associated with slip band features generally do not have a common intersection in the grain boundary below the surface) may suggest that the free surface biases and perhaps even facilitates strain transfer. However, another possibility is that the slip planes continue to be aligned below the surface, as the accommodating system most likely follows the driving force and aligns itself with the incoming slip band trace. This subsurface geometrical accommodation could be accomplished by local cross slip and/or climb. Regardless, these considerations indicate that care must be taken in the interpretation of slip transfer based on surface characterization alone. Unfortunately, it is difficult to characterize the nature of slip or strain transfer in subsurface regions due to the lack of spatial resolution of advanced X-ray techniques. Thus, development of novel approaches to compare subsurface and surface deformation processes are needed to determine how the nature of slip transfer events on the surface differs from the bulk, e.g. [68]. Such difficulties do not negate the significance of deformation phenomena on surface grains, because the surface is often the location of stress concentrations and environmental attack that are life-limiting for structural components.

Because a simple phenomenological model without explicit grain boundary properties was used in the 3D crystal plasticity simulation, the good spatial agreement with experimental measurements suggests that fine-scale details of the slip transfer process may not need to be modeled in order to gain a good estimate of the local driving forces that activate slip. This indicates that correctly simulating the 3D grain geometry may be more important than improving fine-scale aspects of constitutive models. This implies that the models used to obtain largescale statistical information, such as the spatial distribution of 'hot spots', are meaningful if the model is a credible representation of a real material. Nevertheless, methods to introduce fine-scale details of slip transfer into crystal plasticity models are needed to assess the conditions that lead to the nucleation of damage.

Continuing efforts to do nondestructive 3D characterization of deforming 3D microstructure patches will certainly shed more understanding on the nature of slip transfer when com- 
bined with simulations of the experiment. For example, nearfield methods that provide a much more accurate microstructural characterization will enable more accurate simulation of local stress state evolution in complex regions of multi-crystal interfaces, and may enable clear explanations of why rare events happen. The ability to predict rare events will enable spatial prediction of the nucleation of critical damage sites. With such information, it may be possible to predict the failure process in a more deterministic way.

\section{Conclusions}

The analysis of slip transfer occurring in $\mathrm{Ta}$, Ti, and $\mathrm{Ti}$ alloys (serving as examples of body-centered cubic and hexagonal model materials) shows that many observations can be rationalized in terms of the geometrical alignment of activated slip systems in neighboring grains. Because of the difficulty in identifying the driving force for activating all of the observed (and unobserved) deformation systems near grain boundaries, correlations between observations and analytical theories tend to have considerable scatter. Combined characterization and simulation of geometrically accurate patches of real microstructures can provide mutually beneficial information for both the correct interpretation of observations, and identification of mechanisms and phenomena that need to be introduced into models of crystal plasticity. Development of good modeling strategies that track the evolution of slip behavior at and near grain boundaries will increase the ability to predict damage nucleation and evolution in a deterministic and predictive way.

\section{Acknowledgments}

The authors gratefully acknowledge financial support from NSF-Materials World Network (NSF-DMR-1108211, DFG ZA 523/3-1), Max-Planck-Institut für Eisenforschung, DOEBES (DE-FG02-09ER46637), and Sandia National Laboratory. Use of the Advanced Photon Source was supported by the US Department of Energy, Office of Science, Office of Basic Energy Sciences, under Contract No. W-31-109-Eng38. We are grateful for continuous support by D. Raabe during extended summer stays at Max-Planck-Institut für Eisenforschung, Düsseldorf, and the many inspiring discussions with members of his department. We also appreciate microscopy, data collection, and analysis done by undergraduate students Justin Roe and Scott Sutton, and Leyun Wang for unpublished slip transfer data in titanium.

\section{References}

\section{References}

[1] N. Allain-Bonasso, F. Wagner, S. Berbenni, and D. P. Field. A study of the heterogeneity of plastic deformation in IF steel by EBSD. Materials Science and Engineering A, 548:56-63, 2012. doi: 10.1016/j.msea.2012.03.068.

[2] S. Scheriau and R. Pippan. Influence of grain size on orientation changes during plastic deformation. Materials Science and Engineering A, 493 (1-2):48-52, 2008. doi: 10.1016/j.msea.2007.08.092.
[3] C. Rehrl, B. Völker, S. Kleber, T. Antretter, and R. Pippan. Crystal orientation changes: A comparison between a crystal plasticity finite element study and experimental results. Acta Materialia, 60(5):2379-2386, 2012. doi: 10.1016/j.actamat.2011.12.052.

[4] A. Bhattacharyya, E. El-Danaf, S. R. Kalidindi, and R. D. Doherty. Evolution of grain-scale microstructure during large strain simple compression of polycrystalline aluminum with quasi-columnar grains: OIM measurements and numerical simulations. International Journal of Plasticity, 17 (6):861-883, 2001. doi: 10.1016/S0749-6419(00)00072-3.

[5] K-S Cheong and E. P. Busso. Effects of lattice misorientations on strain heterogeneities in FCC polycrystals. Journal of the Mechanics and Physics of Solids, 54(4):671-689, 2006. doi: 10.1016/j.jmps.2005.11.003.

[6] R. A. Lebensohn and C. N. Tomé. A self-consistent anisotropic approach for the simulation of plastic deformation and texture development of polycrystals: Application to zirconium alloys. Acta Metallurgica et Materialia, 41(9):2611-2624, 1993. doi: 10.1016/0956-7151(93)90130-K.

[7] R. A. Lebensohn and C. N. Tomé. A self-consistent viscoplastic model: prediction of rolling textures of anisotropic polycrystals. Materials Science and Engineering A, 175(1-2):71-82, 1994. doi: 10.1016/09215093(94)91047-2.

[8] C. N. Tomé. Self-consistent polycrystal models: a directional compliance criterion to describe grain interactions. Modelling and Simulation in Materials Science and Engineering, 7(5):723-738, 1999.

[9] R. A. Lebensohn. N-site modeling of a 3D viscoplastic polycrystal using Fast Fourier Transform. Acta Materialia, 49(14):2723-2737, 2001. doi: 10.1016/S1359-6454(01)00172-0.

[10] D. Raabe, Z. Zhao, and F. Roters. A finite element method on the basis of texture components for fast predictions of anisotropic forming operations. Steel Res., 72:421-426, 2001.

[11] D. Raabe, M. Sachtleber, Z. Zhao, F. Roters, and S. Zaefferer. Micromechanical and macromechanical effects in grain scale polycrystal plasticity experimentation and simulation. Acta Materialia, 49(17):3433-3441, 2001. doi: 10.1016/S1359-6454(01)00242-7.

[12] S. K. Mishra, P. Pant, K. Narasimhan, A. D. Rollett, and I. Samajdar. On the widths of orientation gradient zones adjacent to grain boundaries. Scripta Materialia, 61(3):273-276, 2009. doi: 10.1016/j.scriptamat.2009.03.062.

[13] L. S. Tóth, Y. Estrin, R. Lapovok, and C. Gu. A model of grain fragmentation based on lattice curvature. Acta Materialia, 58(5):1782-1794, 2010. doi: 10.1016/j.actamat.2009.11.020.

[14] T. R. Bieler, P. D. Nicolaou, and S. L. Semiatin. An experimental and theoretical investigation of the effect of local colony orientations and misorientation on cavitation during hot working of Ti-6Al-4V. Metallurgical and Materials Transactions A, 36(1):129-140, 2005. doi: 10.1007/s11661-005-0145-9.

[15] T. Bieler, R. L. Goetz, and S. L. Semiatin. Anisotropic plasticity and cavity growth during upset forging of Ti-6Al-4V. Materials Science and Engineering: A, 405(1-2):201-213, 2005. doi: 10.1016/j.msea.2005.05.064.

[16] L. Capolungo, D. E. Spearot, M. Cherkaoui, D. L. McDowell, J. Qu, and K. I. Jacob. Dislocation nucleation from bicrystal interfaces and grain boundary ledges: Relationship to nanocrystalline deformation. Journal of the Mechanics and Physics of Solids, 55(11):2300-2327, 2007. doi: 10.1016/j.jmps.2007.04.001.

[17] J. P. Hirth. The Influence of Grain Boundaries on Mechanical Properties. Metallurgical Transactions, 3:3047-3067, 1972.

[18] J. P. Hirth and R. W. Balluffi. On grain boundary dislocations and ledges. Acta Metallurgica, 21(7):929-942, 1973. doi: 10.1016/00016160(73)90150-8.

[19] S-T Shiue. Effect of grain boundary ledge on the dislocation-free zone model of fracture: Transgranular microcrack nucleated from a grain boundary ledge. Journal of Applied Physics, 79(8):3975-, 1996. doi: 10.1063/1.361826.

[20] S-T Shiue and S. Lee. Effect of grain boundary ledges on mode III fracture. Philosophical Magazine A, 67(6):1433-1446, 1993. doi: 10.1080/01418619308225365.

[21] J. D. Clayton and D. L. McDowell. Homogenized finite elastoplasticity and damage: theory and computations. Mechanics of Materials, 36(9): 799-824, 2004.

[22] J. D. Clayton. Dynamic plasticity and fracture in high density polycrystals: constitutive modeling and numerical simulation. Journal 
of the Mechanics and Physics of Solids, 53(2):261-301, 2005. doi: 10.1016/j.jmps.2004.06.009.

[23] J. D. Livingston and B. Chalmers. Multiple slip in bicrystal deformation. Acta Metallurgica, 5(6):322-327, 1957. doi: 10.1016/00016160(57)90044-5.

[24] W. A. T. Clark, R. H. Wagoner, Z. Y. Shen, T. C. Lee, I. M. Robertson, and H. K. Birnbaum. On the criteria for slip transmission across interfaces in polycrystals. Scripta Metallurgica et Materialia, 26(2):203-206, 1992. doi: 10.1016/0956-716X(92)90173-C.

[25] Z. Shen, R. H. Wagoner, and W. A. T. Clark. Dislocation and grain boundary interactions in metals. Acta Metallurgica, 36(12):3231-3242, 1988. doi: 10.1016/0001-6160(88)90058-2.

[26] T. C. Lee, I. M. Robertson, and H. K. Birnbaum. Prediction of slip transfer mechanisms across grain boundaries. Scripta Metallurgica, 23(5):799_ 803, 1989. doi: 10.1016/0036-9748(89)90534-6.

[27] J. Luster and J. M. Morris. Compatibility of deformation in two-phase Ti-Al alloys: Dependence on microstructure and orientation relationships. Metallurgical and Materials Transactions A, 26(7):1745-1756, 1995. doi: 10.1007/BF02670762.

[28] W. M. Ashmawi and M. A. Zikry. Grain-boundary interfaces and void interactions in porous aggregates. Philosophical Magazine, 83(31-34): 3917-3944, 2003. doi: 10.1080/14786430310001599423.

[29] W. M. Ashmawi and M. A. Zikry. Single void morphological and grainboundary effects on overall failure in FCC polycrystalline systems. $\mathrm{Ma}$ terials Science and Engineering A, 343(1-2):126-142, 2003.

[30] G. S. Liu, S. D. House, J. Kacher, M. Tanaka, K. Higashida, and I. M. Robertson. Electron tomography of dislocation structures. Materials Characterization, 87:1-11, 2014. doi: 10.1016/j.matchar.2013.09.016.

[31] J. Kacher and I. M. Robertson. In situ and tomographic analysis of dislocation/grain boundary interactions in $\alpha$-titanium. Philosophical Magazine, pages 1-16, 2014. doi: 10.1080/14786435.2013.868942.

[32] T. R. Bieler, M. A. Crimp, Y. Yang, L. Wang, P. Eisenlohr, D. E. Mason, W. Liu, and G. E. Ice. Strain Heterogeneity and Damage Nucleation at Grain Boundaries during Monotonic Deformation in Commercial Purity Titanium. JOM, 61(12):45-52, 2009. doi: 10.1007/s11837-009-0180-x.

[33] S. Zaefferer, J-C Kuo, Z. Zhao, M. Winning, and D. Raabe. On the influence of the grain boundary misorientation on the plastic deformation of aluminum bicrystals. Acta Materialia, 51(16):4719-4735, 2003. doi: 10.1016/S1359-6454(03)00259-3.

[34] L. C. Lim and R. Raj. The role of residual dislocation arrays in slip induced cavitation, migration and dynamic recrystallization at grain boundaries. Acta Metallurgica, 33(12):2205-2214, 1985. doi: 10.1016/00016160(85)90182-8.

[35] S. Kobayashi, S. Tsurekawa, and T. Watanabe. Grain boundary hardening and triple junction hardening in polycrystalline molybdenum. Acta Materialia, 53(4):1051-1057, 2005. doi: 10.1016/j.actamat.2004.11.002.

[36] B. Liu, P. Eisenlohr, F. Roters, and D. Raabe. Simulation of dislocation penetration through a general low-angle grain boundary. Acta Materialia, 60(13-14):5380-5390, 2012. doi: 10.1016/j.actamat.2012.05.002.

[37] B. A. Simkin, M. A. Crimp, and T. R. Bieler. A factor to predict microcrack nucleation at $\gamma-\gamma$ grain boundaries in TiAl. Scripta Materialia, 49 (2):149-154, 2003. doi: 10.1016/S1359-6462(03)00216-1.

[38] L. Wang, Y. Yang, P. Eisenlohr, T. R. Bieler, M. A. Crimp, and D. E. Mason. Twin Nucleation by Slip Transfer across Grain Boundaries in Commercial Purity Titanium. Metallurgical and Materials Transactions A, 41(2):421-430, 2010. doi: 10.1007/s11661-009-0097-6.

[39] Y. Yang, L. Wang, T. Bieler, P. Eisenlohr, and M. Crimp. Quantitative Atomic Force Microscopy Characterization and Crystal Plasticity Finite Element Modeling of Heterogeneous Deformation in Commercial Purity Titanium. Metallurgical and Materials Transactions A, 42(3):636-644, 2011. doi: 10.1007/s11661-010-0475-0.

[40] H. Li, C. J. Boehlert, T. R. Bieler, and M. A. Crimp. Analysis of slip activity and heterogeneous deformation in tension and tension-creep of $\mathrm{Ti}-$ 5Al-2.5Sn (wt \%) using in-situ SEM experiments. Philosophical Magazine, 92(23):2923-2946, 2012. doi: 10.1080/14786435.2012.682174.

[41] H. Li, D. E. Mason, Y. Yang, T. R. Bieler, M. A. Crimp, and C. J. Boehlert. Comparison of the deformation behaviour of commercially pure titanium and Ti-5Al-2.5Sn(wt.\%) at 296 and 728 K. Philosophical Magazine, 93 (21):2875-2895, 2013. doi: 10.1080/14786435.2013.791752.

[42] H. J. Bunge. Texture Analysis in Materials Science. Butterworths, London, 1982.
[43] P. Eisenlohr, F. Roters, M. Diehl, C. Kords, and P. Shanthraj. DAMASK Web page. 2014. URL http://damask.mpie.de.

[44] B. C. Larson, W. Yang, G. E. Ice, J. D. Budai, and J. Z. Tischler. Threedimensional X-ray structural microscopy with submicrometre resolution. Nature, 415(6874):887-890, 2002. doi: 10.1038/415887a.

[45] T. R. Bieler, L. Wang, A. J. Beaudoin, P. Kenesei, and U. Lienert. In Situ Characterization of Twin Nucleation in Pure Ti Using 3D-XRD. Metallurgical and Materials Transactions A, 45(1):109-122, 2014. doi: 10.1007/s11661-013-2082-3.

[46] T. R. Bieler, S. C. Sutton, B. E. Dunlap, Z. A. Keith, P. Eisenlohr, M. A. Crimp, and B. L. Boyce. Grain Boundary Responses to Heterogeneous Deformation in Tantalum Polycrystals. JOM, 66(1):121-128, 2014. doi: 10.1007/s11837-013-0821-y.

[47] B. A. Simkin, B. C. Ng, M. A. Crimp, and T. R. Bieler. Crack opening due to deformation twin shear at grain boundaries in near- $\gamma$ TiAl. Intermetallics, 15(1):55-60, 2007. doi: 10.1016/j.intermet.2006.03.005.

[48] T. R. Bieler, P. Eisenlohr, F. Roters, D. Kumar, D. E. Mason, M. A. Crimp, and D. Raabe. The role of heterogeneous deformation on damage nucleation at grain boundaries in single phase metals. International Journal of Plasticity, 25(9):1655-1683, 2009. doi: 10.1016/j.ijplas.2008.09.002.

[49] D. Kumar, T. R. Bieler, P. Eisenlohr, D. E. Mason, M. A. Crimp, F. Roters, and D. Raabe. On Predicting Nucleation of Microcracks Due to SlipTwin Interactions at Grain Boundaries in Duplex Near $\gamma$-TiAl. Journal of Engineering Materials and Technology, 130(2):021012-12, 2008. doi: $10.1115 / 1.2841620$

[50] A. Fallahi, D. E. Mason, D. Kumar, T. R. Bieler, and M. A. Crimp. The effect of grain boundary normal on predicting microcrack nucleation using fracture initiation parameters in duplex TiAl. Materials Science and Engineering A, 432(1-2):281-291, 2006.

[51] M. A. Morris. Dislocation configurations in two phase TiAl alloys. II. Structures after compression. Philosophical Magazine A, 68(2):259-278, 1993. doi: 10.1080/01418619308221204.

[52] F. P. E. Dunne, D. Rugg, and A. Walker. Lengthscale-dependent, elastically anisotropic, physically-based hep crystal plasticity: Application to cold-dwell fatigue in Ti alloys. International Journal of Plasticity, 23(6): 1061-1083, 2007. doi: 10.1016/j.ijplas.2006.10.013.

[53] A. L. Pilchak, R. E. A. Williams, and J. C. Williams. Crystallography of Fatigue Crack Initiation and Growth in Fully Lamellar Ti-6Al-4V. Metallurgical and Materials Transactions A, 41(1):106-124, 2010. doi: 10.1007/s11661-009-0064-2.

[54] J. W. Foltz, B. Welk, P. C. Collins, H. L. Fraser, and J. C. Williams. Formation of Grain Boundary $\alpha$ in $\beta$ Ti Alloys: Its Role in Deformation and Fracture Behavior of These Alloys. Metallurgical and Materials Transactions A, 42(3):645-650, 2011. doi: 10.1007/s11661-010-0322-3.

[55] P. Chen, H. Ghassemi-Armaki, S. Kumar, A. Bower, S. Bhat, and $\mathrm{S}$. Sadagopan. Microscale-calibrated modeling of the deformation response of dual-phase steels. Acta Materialia, 65:133-149, 2014. ISSN 1359-6454. doi: 10.1016/j.actamat.2013.11.036.

[56] L. Wang, P. Eisenlohr, Y. Yang, T. R. Bieler, and M. A. Crimp. Nucleation of paired twins at grain boundaries in titanium. Scripta Materialia, 63: 827-830, 2010. doi: 10.1016/j.scriptamat.2010.06.027.

[57] L. Wang, R. I. Barabash, Y. Yang, T. R. Bieler, M. A. Crimp, P. Eisenlohr, W. Liu, and G. E. Ice. Experimental Characterization and Crystal Plasticity Modeling of Heterogeneous Deformation in Polycrystalline $\alpha$-Ti. Metallurgical and Materials Transactions A, 42(3):626-635, 2011. doi: 10.1007/s11661-010-0249-8.

[58] L. Wang, R. Barabash, T. Bieler, W. Liu, and P. Eisenlohr. Study of $\left\{\begin{array}{llll}1 & 1 & \overline{2} & 1\end{array}\right\}$ Twinning in $\alpha$-Ti by EBSD and Laue Microdiffraction. Metallurgical and Materials Transactions A, 44(8):3664-3674, 2013. doi: 10.1007/s11661-013-1714-y.

[59] J. J. Fundenberger, M. J. Philippe, F. Wagner, and C. Esling. Modelling and prediction of mechanical properties for materials with hexagonal symmetry (zinc, titanium and zirconium alloys). Acta Materialia, 45 (10):4041-4055, 1997. doi: 10.1016/S1359-6454(97)00099-2.

[60] X. Tan, H. Gu, C. Laird, and N. D. H. Munroe. Cyclic deformation behavior of high-purity titanium single crystals: Part I. Orientation dependence of stress-strain response. Metallurgical and Materials Transactions A, 29 (2):507-512, 1998. doi: 10.1007/s11661-998-0131-0.

[61] X. Tan, H. Guo, H. Gu, C. Laird, and N. D. H. Munroe. Cyclic deformation behavior of high-purity titanium single crystals: Part II. Microstructure and mechanism. Metallurgical and Materials Transactions A, 29(2): 
513-518, 1998. doi: 10.1007/s11661-998-0132-z.

[62] M. H. Yoo. Slip, Twinning, and Fracture in Hexagonal Close-Packed Metals. Met. Trans. A, 12A:409-415, 1981.

[63] S. Zaefferer. A study of active deformation systems in titanium alloys: dependence on alloy composition and correlation with deformation texture. Materials Science and Engineering A, 344(1-2):20-30, 2003. doi: 10.1016/S0921-5093(02)00421-5.

[64] F. Bridier, P. Villechaise, and J. Mendez. Analysis of the different slip systems activated by tension in a $\alpha / \beta$ titanium alloy in relation with local crystallographic orientation. Acta Materialia, 53(3):555-567, 2005. doi: 10.1016/j.actamat.2004.09.040.

[65] M. R. Barnett, M. D. Nave, and A. Ghaderi. Yield point elongation due to twinning in a magnesium alloy. Acta Materialia, 60(4):1433-1443, 2012 doi: 10.1016/j.actamat.2011.11.022.

[66] R. Xin, C. Guo, Z. Xu, G. Liu, X. Huang, and Q. Liu. Characteristics of

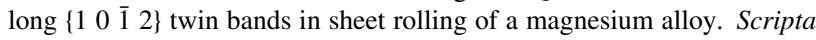
Materialia, 74:96-99, 2014. doi: 10.1016/j.scriptamat.2013.11.008.

[67] C. Guo, R. Xin, C. Ding, B. Song, and Q. Liu. Understanding of variant selection and twin patterns in compressed $\mathrm{Mg}$ alloy sheets via combined analysis of Schmid factor and strain compatibility factor. Materials Science and Engineering: A, 609:92-101, 2014. doi: 10.1016/j.msea.2014.04.103.

[68] A. Fernández, A. Jérusalem, I. Gutiérrez-Urrutia, and M. T. PérezPrado. Three-dimensional investigation of grain boundary-twin interactions in a Mg AZ31 alloy by electron backscatter diffraction and continuum modeling. Acta Materialia, 61(20):7679-7692, 2013. doi: 10.1016/j.actamat.2013.09.005.

[69] L. Margulies, T. Lorentzen, H. F. Poulsen, and T. Leffers. Strain tensor development in a single grain in the bulk of a polycrystal under loading. Acta Materialia, 50(7):1771-1779, 2002. doi: 10.1016/S13596454(02)00028-9.

[70] T. Ungár, G. Ribárik, L. Balogh, A. A. Salem, S. L. Semiatin, and G. B. M. Vaughan. Burgers vector population, dislocation types and dislocation densities in single grains extracted from a polycrystalline commercial-purity Ti specimen by X-ray line-profile analysis. Scripta Materialia, 63(1):69-72, 2010. doi: 10.1016/j.scriptamat.2010.03.016.

[71] H. Abdolvand and M. R. Daymond. Multi-scale modeling and experimental study of twin inception and propagation in hexagonal close-packed materials using a crystal plasticity finite element approach-Part I: Average behavior. Journal of the Mechanics and Physics of Solids, 61(3): 783-802, 2013. doi: 10.1016/j.jmps.2012.10.013.

[72] J. R. Seal, M. A. Crimp, T. R. Bieler, and C. J. Boehlert. Analysis of slip transfer and deformation behavior across the $\alpha / \beta$ interface in Ti-5Al2.5Sn (wt.\%) with an equiaxed microstructure. Materials Science and Engineering A, 552:61-68, 2012. doi: 10.1016/j.msea.2012.04.114. 